\title{
The Fourier Singular Complement Method for the Poisson problem. Part II: axisymmetric domains
}

\author{
P. Ciarlet, Jr, ${ }^{1} \quad$ B. Jung, ${ }^{2} \quad$ S. Kaddouri, ${ }^{3} \quad$ S. Labrunie, ${ }^{4}$ J. Zou ${ }^{5}$
}

\begin{abstract}
This paper is the second part of a threefold article, aimed at solving numerically the Poisson problem in three-dimensional prismatic or axisymmetric domains. In the first part of this series, the Fourier Singular Complement Method was introduced and analysed, in prismatic domains. In this second part, the FSCM is studied in axisymmetric domains with conical vertices, whereas, in the third part, implementation issues, numerical tests and comparisons with other methods are carried out. The method is based on a Fourier expansion in the direction parallel to the reentrant edges of the domain, and on an improved variant of the Singular Complement Method in the 2D section perpendicular to those edges. Neither refinements near the reentrant edges or vertices of the domain, nor cut-off functions are required in the computations to achieve an optimal convergence order in terms of the mesh size and the number of Fourier modes used.
\end{abstract}

Date of this version: July 7, 2005

\section{Introduction}

The Singular Complement Method (SCM) was originally introduced by Assous et al. [6, 7], for the 2D static or instationary Maxwell equations without charges. It was then extended [4, 5] to the fully axisymmetric case, i.e. axisymmetric domains and data, with or without charges. The SCM has been extended in [1] to the 2D Poisson problem. As noted in [12, further extensions to the 2D heat or wave equations, or to similar problems with piecewise constant coefficients, can be obtained easily. Methodologically speaking, the SCM consists in adding some singular test functions to the usual $\mathbb{P}_{1}$ Lagrange FEM so that it recovers the optimal $H^{1}$-convergence rate, even in non-convex domains. In the fully axisymmetric case, one may simply add one singular test function per reentrant edge, and one per conical vertex of sufficiently large aperture.

\footnotetext{
${ }^{1}$ ENSTA \& CNRS UMR 2706, 32, boulevard Victor, 75739 Paris Cedex 15, France. This author was supported in part by France/Hong Kong Joint Research Programme.

${ }^{2}$ Department of Mathematics, Chemnitz University of Technology, D-09107 Chemnitz, Germany. This author was supported by DGA/DSP-ENSTA 00.60.075.00.470.75.01 Research Programme.

${ }^{3}$ ENSTA \& CNRS UMR 2706, 32, boulevard Victor, 75739 Paris Cedex 15, France.

${ }^{4}$ IECN, Université Henri Poincaré Nancy I \& INRIA (projet CALVI), 54506 Vandœuvre-lès-Nancy cedex, France.

${ }^{5}$ Department of Mathematics, The Chinese University of Hong Kong, Shatin, N.T., Hong Kong. The work of this author was fully supported by Hong Kong RGC grants (Project no. 403403 and CUHK4048/02P).
} 
There exist a couple of numerical methods in the literature for accurately solving 2D Poisson problems in non-convex domains. The SCM is clearly different from (anisotropic) mesh refinement techniques [20, 16, and can be applied efficiently to instationary problems (see Remark 4.1 of [12]), since it does not need the refinements of the mesh and thus large time steps may be allowed. However the anisotropic mesh refinement methods have one advantage: they require only a partial knowledge of the most singular part of the solution.

The numerical solution of 3D singular Poisson problems is quite different from the 2D case, and much more difficult. This is a relatively new field of research: most approaches rely on anisotropic mesh refinement, see for instance [16, 17] and Refs. therein. To our knowledge, this series of papers is the first attempt to generalize the SCM for three-dimensional singular Poisson problems.

The rest of the paper is organised as follows. In the next Section, we define the geometry of the axisymmetric domain $\Omega$, and the suitable framework for the study of the Poisson problem in $\Omega$ using a Fourier expansion with respect to the rotational angle $\theta$, namely, weighted Sobolev spaces over the meridian section $\omega$. This suggests a framework for building the Fourier Singular Complement Method (FSCM) for accurately solving the Poisson problem, using a Fourier expansion in $\theta$, and an improved variant of the Singular Complement Method 11] in $\omega$. In Section 3, we study theoretically this variant of the SCM, based on a regular-singular splitting of the solution $u^{k}$ to the $2 \mathrm{D}$ problem (76). The main feature of the splitting is that it is chosen independently of the Fourier index $k$ as soon as $|k| \geq 2$; this independence is important, and very helpful, from the computational point of view. Section 4 presents a few results of finite element theory in the weighted Sobolev spaces. In Section [5 the SCM is considered from a numerical point of view, to approximate $u^{k}$ accurately, vi $\bar{a}$ the discretization of the splitting. In the Section [6, we build the numerical algorithms which define the FSCM, and we show that it has the optimal convergence of order $O\left(h+N^{-1}\right)$, where $h$ is the $2 \mathrm{D}$ mesh size and $N$ is the number of Fourier modes used.

\section{Poisson problem in axisymmetric domains}

\subsection{Geometric setting and notations}

In this article, we consider an axisymmetric domain $\Omega$, ggenerated by the rotation of a polygon $\omega$ around one of its sides, denoted $\gamma_{a}$. The boundary of $\omega$ is hence $\partial \omega=\gamma_{a} \cup \gamma_{b}$, where $\gamma_{b}$ generates the boundary $\Gamma$ of $\Omega$. Thus, $\Omega$ can be described as:

$$
\Omega=\omega \times \mathbf{S}^{1} \cup \gamma_{a} .
$$

The natural cylindrical coordinates will be denoted by $(r, \theta, z)$. The geometrical singularities that may occur on $\Gamma$ are circular edges and conical vertices, which correspond to off-axis corners of $\gamma_{b}$ and to its extremities. Figure 1 precises the various notations associated to these singularities; a more complete description of the geometry of $\omega$ can be found in [3, 4]. 

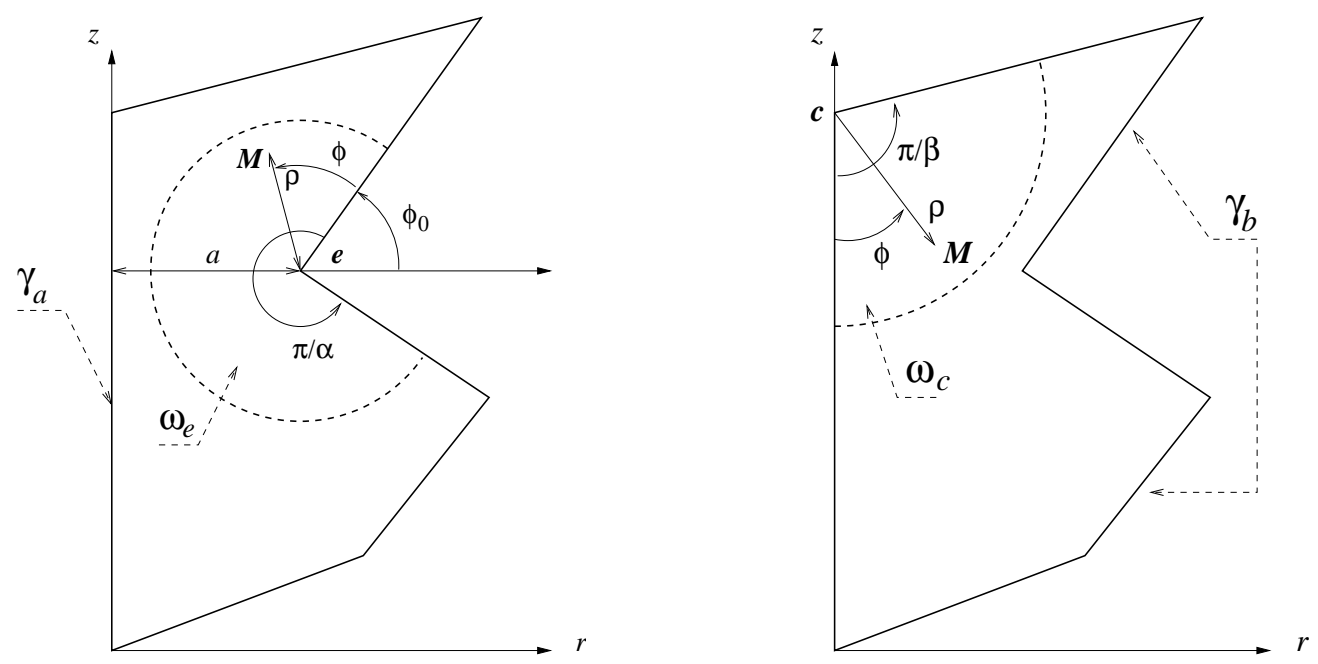

Figure 1: Notations for the geometrical singularities; $\boldsymbol{e}$ : reentrant edge; $\boldsymbol{c}$ : conical vertex.

The problem under consideration is once more the homogeneous Dirichlet problem: Find $u \in \stackrel{\circ}{H}^{1}(\Omega)$ such that

$$
-\Delta u=f \text { in } \Omega, \quad u=0 \text { on } \Gamma,
$$

with $f \in L^{2}(\Omega)$. Non-homogeneous Dirichlet boundary conditions, or (non-)homogeneous Neumann boundary conditions can be handled in exactly the same manner.

As will appear in the sequel, the problem (2) will be singular, i.e. its solution will generically not be in $H^{2}(\Omega)$ - as it would be the case in a regular or convex domainiff there are reentrant edges or sharp vertices in $\Gamma$. Sharp vertices are defined by the condition (see Figure 1):

$$
\nu^{c}<\frac{1}{2}, \quad \text { where: } \nu^{c} \stackrel{\text { def }}{=} \min \left\{\nu>0: P_{\nu}\left(\cos \frac{\pi}{\beta}\right)=0\right\}
$$

and $P_{\nu}$ denotes the Legendre function. This is satisfied iff $\pi / \beta>\pi / \beta_{\star} \simeq 130^{\circ} 48^{\prime}$. From now on, we shall assume that there is exactly one reentrant edge $\boldsymbol{e}$ (of aperture $\pi / \alpha$, with $1 / 2<\alpha<1$ ) and one sharp vertex $\boldsymbol{c}$, and we shall omit the superscript $c$ in $\nu^{c}$.

Other notations. We denote by $r_{\max }$ the supremum of the coordinate $r$ on $\omega$, and by $\alpha_{0}$ and $\alpha_{1}$ two fixed numbers such that

$$
1 / 2<\alpha_{0}<\alpha \text { and } 1 / 2<\alpha_{1}<\min (\alpha, \nu+1 / 2) .
$$

We also introduce 2D neighbourhoods $\omega_{e}$ and $\omega_{c}$ of $\boldsymbol{e}$ and $\boldsymbol{c}$ respectively. They stay away from all sides of $\partial \omega$ except the two ones that meet at the relevant corner. To them we associate cutoff functions denoted $\eta(\rho)$, which vanish outside $\omega_{e}$ or $\omega_{c}$ and depend only on the distance to the corner. 


\subsection{Fourier expansions}

The functions defined on $\Omega$ will be characterised through their Fourier series in $\theta$, the coefficients of which are functions defined on $\omega$, viz.

$$
f(r, \theta, z)=\frac{1}{\sqrt{2 \pi}} \sum_{k=-\infty}^{+\infty} f^{k}(r, z) \mathrm{e}^{\mathrm{i} k \theta},
$$

and the truncated Fourier expansion of $f$ at order $N$ is:

$$
f^{[N]}(r, \theta, z)=\frac{1}{\sqrt{2 \pi}} \sum_{k=-N}^{N} f^{k}(r, z) \mathrm{e}^{\mathrm{i} k \theta} .
$$

The regularity of the function $f$ in the scale $H^{s}(\Omega)$ can be characterised by that of the $\left(f^{k}\right)_{k \in \mathbb{Z}}$ in certain spaces of functions defined over $\omega$ [10, $\S \S I I .1$ to II.3], namely:

$$
f \in H^{s}(\Omega), s \geq 0 \Longleftrightarrow \forall k \in \mathbb{Z}, f^{k} \in H_{(k)}^{s}(\omega),
$$

where the $H_{(k)}^{s}(\omega)$ are defined in turn with the help of two different types of weighted Sobolev spaces. We shall now give these definitions for the values of $s$ and $k$ chiefly needed in this article. The notations for the various spaces are the same as in [10, where the interested reader can find the proofs and the most general versions of the subsequent statements.

First, for any $\tau \in \mathbb{R}$ we consider the weighted Lebesgue space

$$
L_{\tau}^{2}(\omega) \stackrel{\text { def }}{=}\left\{w \text { measurable on } \omega: \iint_{\omega}|w(r, z)|^{2} r^{\tau} \mathrm{d} r \mathrm{~d} z<\infty\right\} .
$$

This space, as well as all the spaces introduced in this article, is a Hermitian space of functions with complex values. The scale $\left(H_{\tau}^{s}(\omega)\right)_{s \geq 0}$ is the canonical Sobolev scale built upon $L_{\tau}^{2}(\omega)$, defined for $s \in \mathbb{N}$ as:

$$
H_{\tau}^{s}(\omega) \stackrel{\text { def }}{=}\left\{w \in L_{\tau}^{2}(\omega): \partial_{r}^{\ell} \partial_{z}^{m} w \in L_{\tau}^{2}(\omega), \forall \ell, m \text { s.t. } 0 \leq \ell+m \leq s\right\},
$$

and by interpolation for $s \notin \mathbb{N}$. We denote by $\|\cdot\|_{s, \tau}$ and $|\cdot|_{s, \tau}$ the canonical norm and semi-norm of $H_{\tau}^{s}(\omega)$.

A prominent role will be played by $L_{1}^{2}(\omega)$; its scalar product is denoted $(\cdot \mid \cdot)$, without any subscript. Upon this space, we build another, dimensionally homogeneous Sobolev scale $\left(V_{1}^{s}(\omega)\right)_{s \geq 0}$, defined as:

$$
V_{1}^{s}(\omega) \stackrel{\text { def }}{=}\left\{w \in H_{1}^{s}(\omega): r^{\ell+m-s} \partial_{r}^{\ell} \partial_{z}^{m} w \in L_{1}^{2}(\omega), \forall \ell, m \text { s.t. } 0 \leq \ell+m \leq\lfloor s\rfloor\right\},
$$

where $\lfloor s\rfloor$ denotes the integral part of $s$. One can check that the general definition reduces to

$$
V_{1}^{s}(\omega)=\left\{w \in H_{1}^{s}(\omega):\left.\partial_{r}^{j} w\right|_{\gamma_{a}}=0, \text { for } 0 \leq j<s-1\right\},
$$

when $s$ is not an integer; while for the first values of $s \in \mathbb{N}$, we have:

$$
V_{1}^{0}(\omega)=L_{1}^{2}(\omega), \quad V_{1}^{1}(\omega)=H_{1}^{1}(\omega) \cap L_{-1}^{2}(\omega), \quad V_{1}^{2}(\omega)=H_{1}^{2}(\omega) \cap H_{-1}^{1}(\omega) .
$$

The canonical norm of $V_{1}^{s}(\omega)$ is denoted by $\|\cdot\|_{s, 1}$; it is equivalent to $|\cdot|_{s, 1}$ except for $s \in \mathbb{N}^{*}$. 
We are now ready to define the most useful spaces of Fourier coefficients.

Lemma 2.1 The spaces $H_{(k)}^{s}(\omega)$, for $s=0,1,2$, are characterised as follows.

$$
\begin{aligned}
& H_{(k)}^{0}(\omega)=L_{1}^{2}(\omega), \forall k, \quad H_{(0)}^{1}(\omega)=H_{1}^{1}(\omega), \quad H_{(k)}^{1}(\omega)=V_{1}^{1}(\omega), \forall|k| \geq 1 ; \\
& H_{(0)}^{2}(\omega)=\left\{w \in H_{1}^{2}(\omega): \partial_{r} w \in L_{-1}^{2}(\omega)\right\}, \quad H_{( \pm 1)}^{2}(\omega)=\left\{w \in H_{1}^{2}(\omega):\left.w\right|_{\gamma_{a}}=0\right\}, \\
& H_{(k)}^{2}(\omega)=V_{1}^{2}(\omega), \forall|k| \geq 2 .
\end{aligned}
$$

The definition for the other values of $s$ will be given when needed.

Remark 2.1 The scales $H_{1}^{s}(\omega), V_{1}^{s}(\omega)$, and $H_{(k)}^{s}(\omega)$ (for any $k$ ) can be extended to negative values of the exponent $s$, by the usual duality procedure with respect to the pivot space, which is $L_{1}^{2}(\omega)$ in all cases.

In order to handle the Dirichlet condition, we introduce the subspaces $\stackrel{\diamond}{H}_{1}^{1}(\omega)$, $\stackrel{\circ}{V}_{1}^{1}(\omega), \stackrel{\circ}{H}_{-1}^{1}(\omega)$ of functions which vanish on $\gamma_{b}$. The difference in the notation is to remind that the functions of $V_{1}^{1}(\omega)$ and $H_{-1}^{1}(\omega) \subset V_{1}^{1}(\omega)$ automatically vanish on $\gamma_{a}$ in a weak sense [18, Prop. 4.1]. This difference is of course important when it comes to discretisation by $\mathbb{P}_{1}$ finite elements.

Similarly to the prismatic case, we introduce the anisotropic Sobolev spaces

$$
\begin{aligned}
& h^{1}(\Omega) \stackrel{\text { def }}{=} H^{1}\left(\mathbf{S}^{1}, L_{1}^{2}(\omega)\right)=\left\{f \in L^{2}(\Omega): \partial_{\theta} f \in L^{2}(\Omega)\right\} ; \\
& h^{2}(\Omega) \stackrel{\text { def }}{=} H^{2}\left(\mathbf{S}^{1}, L_{1}^{2}(\omega)\right)=\left\{f \in h^{1}(\Omega): \partial_{\theta}^{2} f \in L^{2}(\Omega)\right\} ;
\end{aligned}
$$

they are identical to the $H^{0, s}(\Omega)$ of [10, Eq. (II.4.16)], for $s=1,2$.

The next Lemma summarises the completeness results whose proofs can be found in [10, Chapter II] or [15].

Lemma 2.2 The following characterisations hold:

$$
\begin{aligned}
f \in L^{2}(\Omega) & \Longleftrightarrow \forall k \in \mathbb{Z}, f^{k} \in L_{1}^{2}(\omega), \text { and: } \sum_{k=-\infty}^{+\infty}\left\|f^{k}\right\|_{0,1}^{2}<\infty ; \\
f \in h^{s}(\Omega) & \forall k \in \mathbb{Z}, f^{k} \in L_{1}^{2}(\omega), \text { and: } \sum_{k=-\infty}^{+\infty} k^{2 s}\left\|f^{k}\right\|_{0,1}^{2}<\infty ;
\end{aligned}
$$

and the canonical norms of $L^{2}(\Omega)$ and $h^{s}(\Omega)$ are equal to the square roots of these sums. Moreover, defining $V_{(k)}=H_{(k)}^{1}(\omega) \cap \stackrel{\stackrel{\leftrightarrow}{H}}{1}(\omega)$, viz. $\stackrel{\stackrel{\circ}{H}}{1}(\omega)$ for $k=0$ and $\stackrel{\circ}{V_{1}^{1}}(\omega)$ for the other cases, we have:

$$
f \in \stackrel{\circ}{H}^{1}(\Omega) \Longleftrightarrow \forall k \in \mathbb{Z}, f^{k} \in V_{(k)} \text { and }|f|_{H^{1}(\Omega)}^{2}=\sum_{k=-\infty}^{+\infty}\left\|f^{k}\right\|_{(k)}^{2}<\infty,
$$

where the norm $\|w\|_{(k)}^{2}=|w|_{1,1}^{2}+k^{2}\|w\|_{0,-1}^{2}$.

As we did in the prismatic framework, we define the relation operators $\lesssim$ and $\approx$ as follows. $a \lesssim b$ means $a \leq C b$, where $C$ is a constant which depends only on the geometry of the domain $\omega$, and not on the mesh size $h$, the Fourier order $k$, or the data $f$ of the Poisson problem. $a \approx b$ denotes the conjunction of $a \lesssim b$ and $b \lesssim a$. 


\subsection{Singular Poisson problem in 2D}

Denoting by $u^{k}$ and $f^{k}$ the Fourier coefficients of $u$ and $f$ in (2), we see [10, §II.4] that for any $k, u^{k}$ is solution to the following singular Poisson problem in $\omega$ :

Find $u^{k}$ such that

$$
\begin{aligned}
A_{k} u^{k} \stackrel{\text { def }}{=}-\Delta_{k} u^{k} & =f^{k} \text { in } \omega, \quad \text { where: } \quad \Delta_{k} \stackrel{\text { def }}{=} \frac{\partial^{2}}{\partial r^{2}}+\frac{1}{r} \frac{\partial}{\partial r}+\frac{\partial^{2}}{\partial z^{2}}-\frac{k^{2}}{r^{2}}, \\
u^{k} & =0 \text { on } \gamma_{b} .
\end{aligned}
$$

A special role will be played by $\Delta_{0}$, whose values are the traces in a meridian half-plane of the Laplacian of axisymmetric functions. We remark that the operators $\Delta_{k}$ have real coefficients, hence the real and imaginary parts of the solution to (7] [8) correspond to the real and imaginary parts of the data. So, in practice, it will be sufficient to consider problems with real data and solutions.

The variational space associated to (7) 8 is the $V_{(k)}$ defined in Lemma 2.2. The variational formulation reads [10, §II.4.a]:

$$
a_{k}\left(u^{k}, v\right)=\left(f^{k} \mid v\right), \quad \forall v \in V_{(k)},
$$

where $a_{k}$ is now the sesquilinear form defined by the norm $\|\cdot\|_{(k)}$, viz.

$$
a_{k}(u, v)=\iint_{\omega}\left[r \nabla u \cdot \nabla \bar{v}+\frac{k^{2}}{r} u \bar{v}\right] \mathrm{d} \omega .
$$

(In this text, $\nabla$ will always denote the $2 \mathrm{D}$ gradient in the $(r, z)$ plane.)

Like in the prismatic case, we have the following results.

Lemma 2.3 Let $f \in L^{2}(\Omega)$, and $u$ be the solution to (2). Then $\left(u^{[K]}\right)_{K}$ converges to $u$ in $H^{1}(\Omega)$, and $\left(\Delta u^{[K]}\right)_{K}$ converges to $-f$ in $L^{2}(\Omega)$.

Proof: Similar to [12, Corollary 3.1].

$\diamond$

Lemma 2.4 Let $f \in L^{2}(\Omega)$, and $u$ be the solution to (2). Then $\partial_{\theta} u \in H^{1}(\Omega)$.

Proof: One may follow the lines of [12, Corollary 3.2], using the a priori estimates of [15, Thm 4.2] to check that $\left(\partial_{r} \partial_{\theta} u, r^{-1} \partial_{\theta} \partial_{\theta} u, \partial_{z} \partial_{\theta} u\right) \in L^{2}(\Omega)^{3}$.

Besides the variational space, we shall consider, for each Fourier mode $k$ :

- the natural space, which is the one to which $u^{k}$ belongs, i.e. the domain of the operator $A_{k}$ :

$$
D\left(A_{k}\right) \stackrel{\text { def }}{=}\left\{w \in V_{(k)}=H_{(k)}^{1}(\omega) \cap \stackrel{\diamond}{H}_{1}^{1}(\omega): A_{k} w \in L_{1}^{2}(\omega)\right\}
$$

- the regularised space, i.e. the one to which the solution $u^{k}$ would belong if the domain $\Omega$ were regular or convex, namely $H_{(k)}^{2}(\omega) \cap \stackrel{\diamond}{H}_{1}^{1}(\omega)$. 
In [10. Thm II.3.1], it is established that the regularised space no longer depends on $k$ as soon as $|k| \geq 2$; in Theorem 3.2 we will show that the same occurs for the natural space. This suggests that the mode 2 can serve as the "fundamental mode" for the high- $|k|$ modes, just like the mode 0 does in the prismatic case. In contradistinction to the latter, the modes 0 and \pm 1 have to be treated separately, with their own singular functions.

\section{Regular-singular decompositions in the $2 \mathrm{D}$ do- main $\omega$ : theoretical study}

We now establish the regular-singular decompositions, for the various Fourier modes $k$, of the solution $u^{k}$ to (7-8), which will be effectively used in the numerical method. This parallels the work exposed in the companion paper [12, §4].

We shall need the following integration by parts formulae.

Theorem 3.1 For any $u, v \in H_{1}^{1}(\omega)$ such that $\Delta_{0} v \in L_{1}^{2}(\omega)$, there holds:

$$
\iint_{\omega}\left\{u \Delta_{0} v+\nabla u \cdot \nabla v\right\} r \mathrm{~d} \omega=\int_{\gamma_{b}} u \frac{\partial v}{\partial \nu} r \mathrm{~d} \gamma
$$

For any $w \in \stackrel{\circ}{H}_{-1}^{1}(\omega)$ such that $\Delta_{0} w \in L_{1}^{2}(\omega)$, there holds:

$$
\Re \iint_{\omega}-\left\{\frac{\bar{w}}{r^{2}} \Delta_{0} w\right\} r \mathrm{~d} \omega=\|\nabla w\|_{0,-1}^{2}-2\|w\|_{0,-3}^{2} .
$$

Proof: Eq. (11) is the expression, in a meridian half-plane, of the usual Green formula applied to axisymmetric functions. To prove (12), we first note that there holds, in the sense of distributions in $\omega$ :

$$
\nabla w \cdot \nabla\left(\frac{\bar{w}}{r^{2}}\right)=\frac{|\nabla w|^{2}}{r^{2}}-\frac{2 \bar{w}}{r^{3}} \frac{\partial w}{\partial r} .
$$

But $w \in H_{-1}^{1}(\omega)$ implies $w \in L_{-3}^{2}(\omega)$ 3. Lemma 4.9], i.e. $r^{-2} w \in L_{1}^{2}(\omega)$; so the above function is integrable with respect to the measure $r \mathrm{~d} \omega$, and we can apply (11) with $u=r^{-2} \bar{w}$ and $v=w$ :

$$
\begin{aligned}
I_{1} & \stackrel{\text { def }}{=} \iint_{\omega}-\left\{\frac{\bar{w}}{r^{2}} \Delta_{0} w\right\} r \mathrm{~d} \omega=\iint_{\omega} \nabla w \cdot \nabla\left(\frac{\bar{w}}{r^{2}}\right) r \mathrm{~d} \omega \\
& =\iint_{\omega} \frac{|\nabla w|^{2}}{r^{2}} r \mathrm{~d} \omega-2 \iint_{\omega}\left(\frac{1}{r} \frac{\partial w}{\partial r}\right)\left(\frac{\bar{w}}{r^{2}}\right) r \mathrm{~d} \omega \\
& \stackrel{\text { def }}{=}\|\nabla w\|_{0,-1}^{2}-2 I_{2} .
\end{aligned}
$$

Now, we treat $I_{2}$ by the usual integration by parts formula of order one:

$$
\begin{aligned}
\iint_{\omega}\left[\frac{\partial w}{\partial r} \frac{\bar{w}}{r^{2}}+w \frac{\partial}{\partial r} \frac{\bar{w}}{r^{2}}\right] \mathrm{d} \omega=\int_{\gamma} w \frac{\bar{w}}{r^{2}} \nu_{r} \mathrm{~d} \omega & =0 \\
\iint_{\omega}\left[\frac{\partial w}{\partial r} \frac{\bar{w}}{r^{2}}+\frac{w}{r^{2}} \frac{\partial \bar{w}}{\partial r}+|w|^{2}\left(-\frac{2}{r^{3}}\right)\right] \mathrm{d} \omega & =0 \\
2 \Re I_{2}-2\|w\|_{0,-3}^{2} & =0 .
\end{aligned}
$$


Hence, $\Re I_{1}=\|\nabla w\|_{0,-1}^{2}-2\|w\|_{0,-3}^{2}$.

\subsection{Modes $|k| \geq 2$.}

From [10, §II.4], we know the following facts. The solution $u^{k}$ to (17 8) is regular everywhere except in the neighbourhood of the reentrant edge, and it can be written as:

$$
u^{k}=u_{R}^{k}+\lambda_{k} S_{k}^{e}, \quad \text { with: }\left\{\begin{array}{l}
u_{R}^{k} \in H_{(k)}^{2}(\omega) \cap \stackrel{\diamond}{H}_{1}^{1}(\omega)=V_{1}^{2}(\omega) \cap \stackrel{\circ}{V_{1}^{1}}(\omega), \\
S_{k}^{e}(\rho, \phi)=\eta(\rho) \mathrm{e}^{-|k| \rho} \rho^{\alpha} \sin (\alpha \phi) .
\end{array}\right.
$$

As a first consequence, we have the following

Theorem 3.2 Let $w \in D\left(A_{k}\right)$. Then:

- $w$ has a $V_{1}^{2}$ regularity near the axis, hence $w \in \stackrel{\circ}{H}_{-1}^{1}(\omega) \subset L_{-3}^{2}(\omega)$, and both $\Delta_{0} w$ and $r^{-2} w$ are in $L_{1}^{2}(\omega)$.

- $w$ has an $H^{1+\alpha_{0}}$ regularity near the reentrant edge, so its global regularity is $w \in$ $V_{1}^{1+\alpha_{0}}(\omega)$; and there holds: $\|w\|_{1+\alpha_{0}, 1} \leq C(k)\left\|\Delta_{k} w\right\|_{0,1}$.

In close analogy to the orthogonal decomposition of $L^{2}(\omega)$ introduced by Grisvard [14, p. 45], we have:

$$
L_{1}^{2}(\omega)=\Delta_{2}\left[H_{(2)}^{2}(\omega) \cap \stackrel{\circ}{V_{1}^{1}}(\omega)\right] \stackrel{\perp}{\oplus} N_{2},
$$

where $N_{2}$ is a space of singular harmonic functions defined by

$$
N_{2}=\left\{p \in L_{1}^{2}(\omega): \Delta_{2} p=0 \text { in } \omega, p=0 \text { on each side of } \gamma_{b}\right\} .
$$

Here, as well as in the subsequent definitions of $N_{1}$ and $N_{0}$, the boundary condition on the sides of $\gamma_{b}$ is understood in the suitable space, which is the trace in a meridian half-plane of the space $\breve{H}\left(\Gamma_{i}\right)$ defined in [3. Definition 5.4]. Following the same line of proof as in [4, §3], it is not difficult to establish that the dimension of $N_{2}$ is equal to the number of off-axis re-entrant corners in $\omega$, i.e. in our case $\operatorname{dim} N_{2}=1$, and $N_{2}=\operatorname{span}\left\{p_{s}^{2}\right\}$, where $p_{s}^{2}$ can be chosen as:

$$
p_{s}^{2}=\mathrm{S}^{e}+p_{R}^{2}, \quad \text { with: }\left\{\begin{array}{l}
p_{R}^{2} \in \stackrel{\circ}{V_{1}^{1}(\omega)} \\
\mathrm{S}^{e}(\rho, \phi)=\eta(\rho) \rho^{-\alpha} \sin (\alpha \phi)
\end{array}\right.
$$

Similarly to [12, §4], we define $\varphi_{s}^{2}$ as the element of $\stackrel{\circ}{{ }^{1}}(\omega)$ which solves the Poisson problem

$$
-\Delta_{2} \varphi_{s}^{2}=p_{s}^{2} \quad \text { in } \quad \omega .
$$

Then by the decomposition (14), we can split the solution $u^{k}$ to (77 8) as

$$
u^{k}=\tilde{u}^{k}+c_{k} \varphi_{s}^{2}
$$


where $\tilde{u}^{k} \in H_{(2)}^{2}(\omega) \cap \stackrel{\circ}{V_{1}^{1}}(\omega)=H_{(k)}^{2}(\omega) \cap \stackrel{\circ}{H}_{1}^{1}(\omega)$, and is called the regular part of $u^{k}$. How is this decomposition related to (13) ? Applying (13) to $\varphi_{s}^{2}$ itself gives: $\varphi_{s}^{2}=\varphi_{R}^{2}+\delta^{2} S_{2}^{e}$; observing that all the $S_{k}^{e}$ have the same principal part, we deduce $u_{R}^{k}=\tilde{u}^{k}+c_{k} \varphi_{R}^{2}$, and $\lambda_{k}=c_{k} \delta^{2}$. Then, using the orthogonality relation (14) we infer:

$$
\delta^{2}=\frac{\left\|p_{s}^{2}\right\|_{0,1}^{2}}{\left(-\Delta_{2} S_{2}^{e} \mid p_{s}^{2}\right)} .
$$

Calculating this scalar product is rather tedious but can be done using (15) and (11)modified so as to avoid the singularity. We find:

$$
\frac{\lambda_{k}}{c_{k}}=\delta^{2}=\frac{1}{a \pi}\left\|p_{s}^{2}\right\|_{0,1}^{2},
$$

where $a=r(\boldsymbol{e})$ is the distance from the reentrant edge to the axis (see Fig. 1).

The following lemma summarises some a priori estimates on $u^{k}$ and $c_{k}$.

Lemma 3.1 Let $u^{k}$ be the solution to the Poisson problem (78), then we have the following a priori estimates:

$$
\begin{aligned}
k^{2}\left\|u^{k}\right\|_{0,-1} \leq r_{\max }\left\|f^{k}\right\|_{0,1}, \quad k\left|u^{k}\right|_{1,1} & \leq \frac{r_{\max }}{\sqrt{2}}\left\|f^{k}\right\|_{0,1}, \\
\left(k^{2}-2\right)\left\|u^{k}\right\|_{0,-3} \leq\left\|f^{k}\right\|_{0,1}, \quad\left(k^{2}-2\right)^{1 / 2}\left|u^{k}\right|_{1,-1} & \leq \frac{1}{\sqrt{2}}\left\|f^{k}\right\|_{0,1}, \\
\left\|\Delta_{0} u^{k}\right\|_{0,1} & \leq 2\left\|f^{k}\right\|_{0,1}, \\
\left|c_{k}\right| & \lesssim k^{\alpha-1}\left\|f^{k}\right\|_{0,1} \\
\left\|u^{k}\right\|_{1+\alpha_{0}, 1} \approx\left|u^{k}\right|_{1+\alpha_{0}, 1} & \lesssim k^{\alpha_{0}-1}\left\|f^{k}\right\|_{0,1} .
\end{aligned}
$$

Proof: The variational formulation (9) with $v=u^{k}$ gives:

$$
\left|u^{k}\right|_{1,1}^{2}+k^{2}\left\|u^{k}\right\|_{0,-1}^{2} \leq\left\|f^{k}\right\|_{0,1}\left\|u^{k}\right\|_{0,1} \leq r_{\max }\left\|f^{k}\right\|_{0,1}\left\|u^{k}\right\|_{0,-1},
$$

this proves the first estimate in (19). Then applying the Young inequality, we further obtain

$$
\left|u^{k}\right|_{1,1}^{2}+k^{2}\left\|u^{k}\right\|_{0,-1}^{2} \leq \frac{r_{\max }}{2}\left[\frac{r_{\max }}{k^{2}}\left\|f^{k}\right\|_{0,1}^{2}+\frac{k^{2}}{r_{\max }}\left\|u^{k}\right\|_{0,-1}^{2}\right],
$$

which leads to the $H_{1}^{1}$ semi-norm estimate in (19). Similarly, multiplying (7) by $r^{-2} \bar{u}^{k}$ and using (12) yields:

$$
\left|u^{k}\right|_{1,-1}^{2}+\left(k^{2}-2\right)\left\|u^{k}\right\|_{0,-3}^{2} \leq\left\|f^{k}\right\|_{0,1}\left\|u^{k}\right\|_{0,-3},
$$

and we obtain the two estimates in (20) by a similar reasoning. Then (21) immediately follows from $\Delta_{0} u^{k}=f^{k}-k^{2} r^{-2} u^{k}$.

The formula (18) implies: $\left|c_{k}\right| \approx\left|\lambda_{k}\right|$; thus, the estimate (22) is clearly equivalent to: $\left|\lambda_{k}\right| \lesssim k^{\alpha-1}$. This, in turn, can be obtained by following the lines of [14, $\left.\$ 2.5 .2\right]$ or [4, §5.1]. As a matter of fact, the latter reference shows that, away from the axis, the weights in the Sobolev spaces and the exact form of the modified Laplacian under consideration are of no importance. 
Now, setting $f_{R}^{k} \stackrel{\text { def }}{=}-\Delta_{k}\left(u^{k}-c_{k} \varphi_{s}^{2}\right)$, i.e.

$$
f_{R}^{k}=-\Delta_{0} u_{R}^{k}+\frac{k^{2}}{r^{2}} u_{R}^{k},
$$

one concludes, like in the above references, that $\left\|f_{R}^{k}\right\|_{0,1} \lesssim\left\|f^{k}\right\|_{0,1}$. Expanding the squared norm of the equality (24) and using (12) then yields:

$$
\left\|\Delta_{0} u_{R}^{k}\right\|_{0,1}^{2}+2 k^{2}\left|u_{R}^{k}\right|_{1,-1}^{2}+\left(k^{4}-4 k^{2}\right)\left\|u_{R}^{k}\right\|_{0,-3}^{2} \lesssim\left\|f^{k}\right\|_{0,1}^{2} .
$$

On the other hand, there holds: $u_{R}^{k} \in H_{(0)}^{2}(\omega) \cap \stackrel{\leftrightarrow}{H}_{1}^{1}(\omega)$, and within this space the canonical norm of $H_{(0)}^{2}(\omega)$ is equivalent to the norm $\left\|\Delta_{0} w\right\|_{0,1}$ [4, Lemma 4.7]. So, we have both $\left|u_{R}^{k}\right|_{2,1} \lesssim\left\|f^{k}\right\|_{0,1}$ and $\left|u_{R}^{k}\right|_{1,1} \lesssim k^{-1}\left\|f^{k}\right\|_{0,1}$; and we obtain by interpolating in the scale $H_{1}^{s}(\omega)$ that: $\left|u_{R}^{k}\right|_{1+\alpha_{0}, 1} \lesssim k^{\alpha_{0}-1}\left\|f^{k}\right\|_{0,1}$. We then derive (23) by adapting the proof of Lemma 4.1 of [12].

Lemma 3.2 The regular part $\tilde{u}^{k}$ and the singularity coefficient $c_{k}$ in (17) are given as the unique solution of the coupled system:

$$
\begin{aligned}
& a_{k}\left(\tilde{u}^{k}, v\right)+c_{k} a_{k}\left(\varphi_{s}^{2}, v\right)=\left(f^{k} \mid v\right), \quad \forall v \in \stackrel{\circ}{V}_{1}^{1}(\omega), \\
& \left(\left\|p_{s}^{2}\right\|_{0,1}^{2}+\mu\left[\left|\varphi_{s}^{2}\right|_{1,-1}^{2}+2\left\|\varphi_{s}^{2}\right\|_{0,-3}^{2}\right]\right) c_{k}+\mu\left(\tilde{u}^{k} \mid p_{s}^{2}\right)=\left(f^{k} \mid p_{s}^{2}\right),
\end{aligned}
$$

where the symbol $\mu \stackrel{\text { def }}{=} k^{2}-4$. And $\tilde{u}^{k}$ and $c_{k}$ have the following stability estimates:

$$
\left\|\tilde{u}^{k}\right\|_{(k)} \lesssim k\left\|f^{k}\right\|_{0}, \quad\left|c_{k}\right| \leq 2 \frac{\left\|f^{k}\right\|_{0,1}}{\left\|p_{s}^{2}\right\|_{0,1}}, \quad\left\|\tilde{u}^{k}\right\|_{2,1} \lesssim\left\|f^{k}\right\|_{0,1} .
$$

We omit the details of the proof, which is very similar to that of Lemma 4.2 of [12. It makes use of the result: $\left(r^{-2} \varphi_{s}^{2} \mid p_{s}^{2}\right)=\left|\varphi_{s}^{2}\right|_{1,-1}^{2}+2\left\|\varphi_{s}^{2}\right\|_{0,-3}^{2}$, which directly follows from (12) and (16), as $p_{s}^{2}$ and $\varphi_{s}^{2}$ are real. The representation formula for the singularity coefficient is:

$$
c_{k}=\frac{\left(f^{k}-\left(\mu / r^{2}\right) A_{k}^{-1} f^{k} \mid p_{s}^{2}\right)}{\left\|p_{s}^{2}\right\|_{0,1}^{2}} .
$$

The scalar product $\left(r^{-2} A_{k}^{-1} f^{k} \mid p_{s}^{2}\right)$ in (28) is defined thanks to Theorem 3.2. We shall see - and this will be of practical relevance - that it can be written as $\left(A_{k}^{-1} f^{k} \mid r^{-2} p_{s}^{2}\right)$, i.e. $p_{s}^{2} \in L_{-3}^{2}(\omega)$. This is a consequence of the following lemma.

Lemma 3.3 The dual singularity $p_{s}^{2}$ is of $V_{1}^{2}$ regularity near the axis. It admits the following splitting:

$$
p_{s}^{2}=\tilde{p}^{2}+p_{P}^{2}, \quad \tilde{p}^{2} \in V_{1}^{1}(\omega), \quad p_{P}^{2}=\left(\frac{r}{a}\right)^{2} \rho^{-\alpha} \sin (\alpha \phi) .
$$

Similarly, the primal singularity $\varphi_{s}^{2}$ can be represented as:

$$
\varphi_{s}^{2}=\tilde{\varphi}^{2}+\delta^{2} \varphi_{P}^{2}, \quad \tilde{\varphi}^{2} \in V_{1}^{2}(\omega), \quad \varphi_{P}^{2}=\left(\frac{r}{a}\right)^{2} \rho^{\alpha} \sin (\alpha \phi) .
$$


Proof: Let $0<a^{\prime \prime}<a^{\prime}<a$; we consider a cut-off function $\chi$ such that $\chi(r)=1$ for $r \leq a^{\prime \prime}$ and $\chi(r)=0$ for $r \geq a^{\prime}$, as well as the domain $\omega^{\prime}=\left\{\boldsymbol{x} \in \omega: r(\boldsymbol{x})<a^{\prime}\right\}$. This domain has no off-axis reentrant corner (see Figure 11), so there are no singularities of $\Delta_{2}$, either primal or dual, in $\omega^{\prime}$.

As we stay away from the reentrant corner, the splitting (15) shows that $p_{s}^{2} \in$ $V_{1}^{1}\left(\omega^{\prime}\right)$. Thus, $\chi p_{s}^{2} \in V_{1}^{1}\left(\omega^{\prime}\right)$ and it vanishes on $\partial \omega^{\prime}$. Moreover:

$$
\Delta_{2}\left(\chi p_{s}^{2}\right)=\chi \Delta_{2} p_{s}^{2}+\nabla \chi \cdot \nabla p_{s}^{2}+p_{s}^{2} \Delta \chi+\frac{p_{s}^{2}}{r} \frac{\partial \chi}{\partial r} \in L_{1}^{2}\left(\omega^{\prime}\right)
$$

since the first term is identically zero, and the other three are smooth and vanish near the axis. We conclude from Theorem 3.2, and the absence of primal singularities, that $\chi p_{s}^{2} \in V_{1}^{2}\left(\omega^{\prime}\right)$, i.e. $p_{s}^{2}$ is $V_{1}^{2}$ where $\chi=1$.

Now, using (see Figure 10):

$$
\left(\frac{r}{a}\right)^{2}-1=\frac{(r-a)(r+a)}{a^{2}}=\frac{2}{a} \rho \cos \left(\phi+\phi_{0}\right)+\text { h.o.t. }
$$

we remark

$\mathrm{S}^{e}-p_{P}^{2}=\rho^{1-\alpha}\left(g_{1}(\phi)+\right.$ h.o.t. $) \in H^{1}\left(\omega_{e}\right), \quad S_{2}^{e}-\varphi_{P}^{2}=\rho^{1+\alpha}\left(g_{2}(\phi)+\right.$ h.o.t. $) \in H^{2}\left(\omega_{e}\right)$,

since the functions $g_{1,2}(\phi)$ as well as the higher-order terms (h.o.t.) are smooth. Moreover, thanks to the factor $(r / a)^{2}, p_{P}^{2}$ and $\varphi_{P}^{2}$ are of $V_{1}^{2}$ regularity near the axis. The smoothness of these functions in the rest of the domain yields $\mathrm{S}^{e}-p_{P}^{2} \in V_{1}^{1}(\omega)$, $S_{2}^{e}-\varphi_{P}^{2} \in V_{1}^{2}(\omega)$. This proves (29) and (30).

\subsection{Modes $k= \pm 1$.}

As we can see from Lemma 2.1 the variational space is still $\stackrel{\circ}{V}_{1}^{1}(\omega)$; but the regularised space has changed. Once again, the only singularities are located at the reentrant edges. Hence, the solution $u^{k}$ to (77 8 ), with $k= \pm 1$, can be split as:

$$
u^{k}=u_{R}^{k}+\lambda_{k} S_{ \pm 1}^{e}, \quad \text { with: }\left\{\begin{array}{l}
u_{R}^{k} \in H_{( \pm 1)}^{2}(\omega) \cap \stackrel{\circ}{V_{1}^{1}}(\omega)=H_{1}^{2}(\omega) \cap \stackrel{\circ}{V_{1}^{1}}(\omega), \\
S_{ \pm 1}^{e}(\rho, \phi)=\eta(\rho) \mathrm{e}^{-\rho} \rho^{\alpha} \sin (\alpha \phi) .
\end{array}\right.
$$

As a consequence of Theorem 3.2, $\varphi_{s}^{2} \in D\left(A_{1}\right)$, and the decomposition (17) is still valid in this case. However, that singular function belongs to a space which appears too constrained for the modes \pm 1 : it is even better decaying near the axis than the functions of $H_{1}^{2}(\omega) \cap \stackrel{\circ}{V_{1}^{1}}(\omega)$; moreover, this decay is lost in the discretisation by $\mathbb{P}_{1}$ finite elements. So the representation formula (28), though valid at the continuous level with $\mu=-3$, is numerically hardly stable and its use would deteriorate the convergence rate of the SCM. 
So, it is better to use singular functions that are adapted for these modes. Let $p_{s}^{1}$ be a basis of the dual singular space

$$
N_{1}=\left\{p \in L_{1}^{2}(\omega): \Delta_{1} p=0 \text { in } \omega, p=0 \text { on each side of } \gamma_{b}\right\},
$$

and $\varphi_{s}^{1}=A_{1}^{-1} p_{s}^{1}$. These functions were defined and studied in [5, §4.1], and a numerical method was defined. We will introduce below (\$5.4) a slight modification of that method in order to improve the convergence rate. For the moment, we recall that the function $u^{k}$ admits the splitting

$$
u^{k}=\tilde{u}^{k}+c_{k} \varphi_{s}^{1}
$$

where $\tilde{u}^{k} \in H_{(1)}^{2}(\omega) \cap \stackrel{\circ}{H}_{1}^{1}(\omega)=H_{1}^{2}(\omega) \cap \stackrel{\circ}{V}{ }_{1}^{1}(\omega)$. As we are in the "usual" SCM framework [11, we have the simple representation formula

$$
c_{k}=\frac{\left(f^{k} \mid p_{s}^{1}\right)}{\left\|p_{s}^{1}\right\|_{0,1}^{2}}
$$

and the regular part satisfies:

$$
a_{1}\left(\tilde{u}^{k}, v\right)+c_{k} a_{1}\left(\varphi_{s}^{1}, v\right)=\left(f^{k} \mid v\right) \quad \forall v \in \stackrel{\circ}{V_{1}^{1}}(\omega)
$$

From the above considerations easily follow the estimates:

$$
\begin{aligned}
& \left|u^{k}\right|_{1,1} \lesssim\left\|f^{k}\right\|_{0,1}, \quad\left\|u^{k}\right\|_{0,-1} \lesssim\left\|f^{k}\right\|_{0,1}, \quad\left\|\Delta_{0} u^{k}\right\|_{0,1} \lesssim\left\|f^{k}\right\|_{0,1}, \\
& \left|u^{k}\right|_{1+\alpha_{0}, 1} \lesssim\left\|f^{k}\right\|_{0,1}, \quad\left\|\tilde{u}^{k}\right\|_{1,1} \lesssim\left\|f^{k}\right\|_{0,1}, \quad\left|c_{k}\right| \lesssim\left\|f^{k}\right\|_{0,1} .
\end{aligned}
$$

\subsection{Mode $k=0$.}

Now, the variational space is $V_{(0)}=\stackrel{\diamond}{H}_{1}^{1}(\omega)$, and the regularised space is $H_{(0)}^{2}(\omega) \cap V_{(0)}$, with $H_{(0)}^{2}(\omega)$ given by Lemma 2.1] Moreover, there is one singularity per reentrant edge and one per sharp vertex, see [10, §II.4] or [4, §4.4]. The splitting of $u^{0}$ with respect to regularity thus becomes:

$$
u^{0}=u_{R}^{0}+\lambda_{0}^{e} S_{0}^{e}+\lambda_{0}^{c} S_{0}^{c}, \quad \text { with: }\left\{\begin{array}{l}
u_{R}^{0} \in H_{(0)}^{2}(\omega) \cap \stackrel{\diamond}{H}_{1}^{1}(\omega), \\
S_{0}^{e}(\rho, \phi)=\eta(\rho) \rho^{\alpha} \sin (\alpha \phi), \\
S_{0}^{c}(\rho, \phi)=\eta(\rho) \rho^{\nu} P_{\nu}(\cos \phi) .
\end{array}\right.
$$

Once more, there holds: $H_{(0)}^{2}(\omega) \subset H_{(2)}^{2}(\omega)$; yet, once more, attempting to represent the singularity at the edge with the help of the function $\varphi_{s}^{2}$ would imperil the convergence rate of the numerical method. As a consequence, we shall use the "natural" singular functions for this mode (see [4, 5] for details). The dual singular space is

$$
N_{0}=\left\{p \in L_{1}^{2}(\omega): \Delta_{0} p=0 \text { in } \omega, p=0 \text { on each side of } \gamma_{b}\right\} ;
$$


it is of dimension two, with the basis $\left\{p_{s}^{0, e}, p_{s}^{0, c}\right\}$ given by

$$
\left.\begin{array}{l}
p_{s}^{0, e}=\mathrm{S}^{e}+p_{R}^{0, e}, \\
p_{s}^{0, c}=\mathrm{S}^{c}+p_{R}^{0, c},
\end{array}\right\} \quad \text { with: } \quad\left\{\begin{array}{l}
p_{R}^{0, e}, p_{R}^{0, c} \in \stackrel{\diamond}{H_{1}^{1}}(\omega), \\
\mathrm{S}^{c}(\rho, \phi)=\eta(\rho) \rho^{-\nu-1} P_{\nu}(\cos \phi),
\end{array}\right.
$$

and $\mathrm{S}^{e}$ is as in (15). The primal singular functions $\varphi_{s}^{0, j} \in \stackrel{\diamond}{H}_{1}^{1}(\omega)(j=e, c)$ are of course defined as: $\varphi_{s}^{0, j}=A_{0}^{-1} p_{s}^{0, j}$.

Lemma 3.4 The primal singular function $\varphi_{s}^{0, j}$ admits the splitting

$$
\begin{aligned}
\varphi_{s}^{0, j} & =\varphi_{R}^{0, j}+\delta^{0, j} S_{0}^{j}, \quad \text { where }: \varphi_{R}^{0, j} \in H_{(0)}^{2}(\omega) \cap \stackrel{\diamond}{H}_{1}^{1}(\omega), \text { and: } \\
\delta^{0, c} & =\left\|p_{s}^{0, c}\right\|_{0,1}^{2}\left\{(1+2 \nu) \int_{0}^{\pi / \beta} P_{\nu}(\cos \phi)^{2} \sin \phi \mathrm{d} \phi\right\}^{-1}, \\
\delta^{0, e} & =\left\|p_{s}^{0, e}\right\|_{0,1}^{2} /(a \pi) .
\end{aligned}
$$

Equivalently, $p_{s}^{0, j}$ and $\varphi_{s}^{0, j}$ can be represented as

$$
\begin{gathered}
p_{s}^{0, j}=\tilde{p}^{0, j}+p_{P}^{0, j}, \quad \tilde{p}^{0, j} \in H_{1}^{1}(\omega), \quad\left\{\begin{array}{l}
p_{P}^{0, c}=\rho^{-\nu-1} P_{\nu}(\cos \phi), \\
p_{P}^{0, e}=\rho^{-\alpha} \sin (\alpha \phi),
\end{array}\right. \\
\varphi_{s}^{0, j}=\tilde{\varphi}^{0, j}+\delta^{0, j} \varphi_{P}^{0, j}, \quad \tilde{\varphi}^{0, j} \in H_{1}^{2}(\omega), \quad\left\{\begin{array}{l}
\varphi_{P}^{0, c}=\rho^{\nu} P_{\nu}(\cos \phi), \\
\varphi_{P}^{0, e}=\rho^{\alpha} \sin (\alpha \phi) .
\end{array}\right.
\end{gathered}
$$

Proof: Let us examine first the conical singularity: $j=c$. The splitting (37) applied to $\varphi_{s}^{0, c}$ yields: $\varphi_{s}^{0, c}=\varphi_{R}^{0, c}+\delta_{e}^{0, c} S_{0}^{e}+\delta_{c}^{0, c} S_{0}^{c}$. Using the definitions of $p_{s}^{0, c}$ and $\varphi_{s}^{0, c}$ we deduce

$$
\left\|p_{s}^{0, c}\right\|_{0,1}^{2}=\delta_{e}^{0, c}\left(-\Delta_{0} S_{0}^{e} \mid p_{s}^{0, c}\right)+\delta_{c}^{0, c}\left(-\Delta_{0} S_{0}^{c} \mid p_{s}^{0, c}\right) .
$$

Rewriting the first scalar product in (44) as:

$$
\left(-\Delta_{0} S_{0}^{e} \mid p_{s}^{0, c}\right)=\left(-\Delta_{0} S_{0}^{e} \mid p_{R}^{0, c}\right)+\left(-\Delta_{0} S_{0}^{e} \mid \mathrm{S}^{c}\right)
$$

we notice that the second term is zero by a support argument. To evaluate the first term, we remark that $-\Delta_{0} p_{R}^{0, c}=\Delta_{0} \mathrm{~S}^{c}$ vanishing near the conical point, is smooth and belongs to $L_{1}^{2}(\omega)$. So, both $S_{0}^{c}$ and $p_{R}^{0, c}$ are functions in $\stackrel{\diamond}{H}_{1}^{1}(\omega)$ with Laplacian $\Delta_{0}$ in $L_{1}^{2}(\omega)$; and we use (11) twice to obtain:

$$
\left(-\Delta_{0} S_{0}^{e} \mid p_{R}^{0, c}\right)=\left(S_{0}^{e} \mid-\Delta_{0} p_{R}^{0, c}\right)=\left(S_{0}^{e} \mid \Delta_{0} \mathrm{~S}^{c}\right),
$$

which again vanishes by a support argument. Finally, the last scalar product in (44) can be computed e.g. as in [19] to obtain

$$
\left(-\Delta_{0} S_{0}^{c} \mid p_{s}^{0, c}\right)=(1+2 \nu) \int_{0}^{\pi / \beta} P_{\nu}(\cos \phi)^{2} \sin \phi \mathrm{d} \phi
$$

and (3940) are proven. This immediately yields (42 43). Similar computations are carried out for the edge singularity; $\delta^{0, e}$ is computed like $\delta^{2}$ above. 
Lemma 3.5 The solution to $-\Delta_{0} u^{0}=f^{0}$ can be represented as

$$
u^{0}=\tilde{u}^{0}+c_{0, e} \varphi_{s}^{0, e}+c_{0, c} \varphi_{s}^{0, c}, \quad \text { where: } \tilde{u}^{0} \in H_{(0)}^{2}(\omega) \cap \stackrel{\diamond}{H}_{1}^{1}(\omega) .
$$

The $c_{0, j}$ are given by the representation formulae:

$$
c_{0, j}=\frac{\left(f^{0} \mid p_{s}^{0, j}\right)}{\left\|p_{s}^{0, j}\right\|_{0,1}^{2}} .
$$

Proof. As the space of singularities is of dimension two, it is enough to exhibit two linearly independent functions to have a basis. This is obviously the case of $\varphi_{s}^{0, e}$ and $\varphi_{s}^{0, c}$, which proves (45). Taking the Laplacian $-\Delta_{0}$ of this equality and the scalar product by $p_{s}^{0, c}$ yields, thanks to the orthogonality property:

$$
\left(f^{0} \mid p_{s}^{0, c}\right)=c_{0, e}\left(-\Delta_{0} \varphi_{s}^{0, e} \mid p_{s}^{0, c}\right)+c_{0, c}\left\|p_{s}^{0, c}\right\|_{0,1}^{2} .
$$

Then, using the decomposition (39), we obtain: $\left(-\Delta_{0} \varphi_{s}^{0, e} \mid p_{s}^{0, c}\right)=\delta^{0, e}\left(-\Delta_{0} S_{0}^{e} \mid p_{s}^{0, c}\right)$, which is zero as seen in the proof of Lemma 3.4. Hence (46) for $j=c$; the case $j=e$ is treated similarly.

Let us state without proof the elliptic equation satisfied by $\tilde{u}^{0}$ :

$$
a_{0}\left(\tilde{u}^{0}, v\right)+c_{0, e} a_{0}\left(\varphi_{s}^{0, e}, v\right)+c_{0, c} a_{0}\left(\varphi_{s}^{0, c}, v\right)=\left(f^{0} \mid v\right), \quad \forall v \in \stackrel{\leftrightarrow}{H}_{1}^{1}(\omega),
$$

and the stability estimates on the various terms in (45):

$$
\begin{array}{ll}
\left|u^{0}\right|_{1,1} \lesssim\left\|f^{0}\right\|_{0,1}, \quad & \left|u^{0}\right|_{1+\alpha_{1}, 1} \lesssim\left\|f^{0}\right\|_{0,1}, \\
\left|\tilde{u}^{0}\right|_{1,1} \lesssim\left\|f^{0}\right\|_{0,1}, & \left|c_{0, j}\right| \lesssim\left\|f^{0}\right\|_{0,1} .
\end{array}
$$

\section{Interpolation and projection operators}

We consider a regular triangulation of the domain $\omega$, with mesh size $h$. The space spanned by $\mathbb{P}_{1}$ finite elements on this triangulation is denoted $V^{h}$; the subspace of functions which vanish on the whole of $\partial \omega$ is $V_{\circ}^{h}=V^{h} \cap \stackrel{\circ}{V_{1}^{1}}(\omega)$; while $V_{\diamond}^{h}=V^{h} \cap \stackrel{\diamond}{H}_{1}^{1}(\omega)$ is the subspace of functions which vanish only on $\gamma_{b}$. We introduce the usual Lagrange interpolation operator $\Pi_{h}$ as well as the weighted Clément operator $P_{h}$. The latteridentical to the operator called $\widetilde{\Pi}_{h}^{0}$ in $[8, \S 4]$-is a local projection operator onto $\mathbb{P}_{1}$ in the $L_{1}^{2}$ sense, which does not take into account the nodes of the triangulation which stand on $\partial \omega$. Hence, it maps $\stackrel{\circ}{V}_{1}^{1}(\omega)$ onto $V_{\circ}^{h}$.

We now prove a few results on these operators, in the framework of weighted Sobolev spaces of fractional order. We begin by a useful density lemma.

Lemma $4.1 V_{1}^{2}(\omega) \cap \stackrel{\circ}{V} \frac{1}{1}(\omega)$ is dense within $V_{1}^{1+\alpha_{0}}(\omega) \cap \stackrel{\circ}{V_{1}^{1}}(\omega)=H_{1}^{1+\alpha_{0}}(\omega) \cap \stackrel{\circ}{V}{ }_{1}^{1}(\omega)$.

Proof: $\quad$ Let $w \in V_{1}^{1+\alpha_{0}}(\omega) \cap \stackrel{\circ}{V_{1}^{1}}(\omega)$ and $\varepsilon>0$. The construction of $\tilde{w} \in V_{1}^{2}(\omega) \cap$ $\stackrel{\circ}{V}_{1}^{1}(\omega)$ such that $\|w-\tilde{w}\|_{1+\alpha_{0}, 1} \leq \varepsilon$ is decomposed into two steps. The first one will not be effectively used in this article, since we do not consider arbitrary functions in $V_{1}^{1+\alpha_{0}}(\omega) \cap \stackrel{\circ}{V}{ }_{1}^{1}(\omega)$, but only those which belong to $D\left(A_{2}\right)$. 
Step 1: From $H_{1}^{1+\alpha_{0}}(\omega) \cap \stackrel{\circ}{V_{1}^{1}}(\omega)$ To $D\left(A_{2}\right)$. From [10, Thm II.4.8], we know that $A_{k}^{-1}$ is an isomorphism from $H_{(k)}^{s-1}(\omega)$ to $H_{(k)}^{s+1}(\omega) \cap \stackrel{\diamond}{H}_{1}^{1}(\omega)$ for $s<\alpha$. Let $C(s, k)$ be the norm of this operator. Setting $g=-\Delta_{2} w \in H_{(2)}^{\alpha_{0}-1}(\omega)=H_{1}^{\alpha_{0}-1}(\omega)$, we use the density of $H_{1}^{0}(\omega)$ within $H_{1}^{\alpha_{0}-1}(\omega)$ to construct $g^{\prime} \in L_{1}^{2}(\omega)$ such that $\left\|g-g^{\prime}\right\|_{1-\alpha_{0}, 1}<$ $\varepsilon /\left(2 C\left(\alpha_{0}, 2\right)\right)$. Then $w^{\prime} \stackrel{\text { def }}{=} A_{2}^{-1} g^{\prime} \in D\left(A_{2}\right)$ and satisfies $\left\|w-w^{\prime}\right\|_{1+\alpha_{0}, 1}<\varepsilon / 2$.

Step 2: From $D\left(A_{2}\right)$ то $V_{1}^{2}(\omega) \cap \stackrel{\circ}{V_{1}^{1}}(\omega)$. There remains to find $\tilde{w} \in V_{1}^{2}(\omega) \cap \stackrel{\circ}{V_{1}^{1}}(\omega)$ such that $\left\|w^{\prime}-\tilde{w}\right\|_{1+\alpha_{0}, 1}<\varepsilon / 2$. Since $D\left(A_{2}\right)=V_{1}^{2}(\omega) \cap \stackrel{\circ}{V}_{1}^{1}(\omega) \oplus \operatorname{span} S_{2}^{e}$, this is obviously equivalent to find $\tilde{S} \in V_{1}^{2}(\omega) \cap \stackrel{\circ}{V_{1}^{1}}(\omega)$ such that $\left\|S_{2}^{e}-\tilde{S}\right\|_{1+\alpha_{0}, 1} \leq \varepsilon^{\prime}$, for arbitrary $\varepsilon^{\prime}$.

We claim that $\tilde{S}=S_{2}^{e}-S_{k}^{e}$ does the job for $k$ large enough. As a matter of fact, $S_{2}^{e}-S_{k}^{e}=\rho^{1+\alpha} \sin (\alpha \phi)+$ h.o.t. $\in H^{2}\left(\omega_{e}\right) \cap \stackrel{\circ}{H^{1}}\left(\omega_{e}\right)$; thanks to the cutoff function $\eta$, this gives $S_{2}^{e}-S_{k}^{e} \in V_{1}^{2}(\omega) \cap \stackrel{\circ}{V_{1}^{1}}(\omega)$. Then, it is enough to check that

$$
S_{k}^{e} \rightarrow 0 \text { strongly in } H^{1+\alpha_{0}}\left(\omega_{e}\right) \text { as } k \rightarrow+\infty .
$$

This is done like in [14, Lemma 5.3.3], by using the Sobolev imbedding $H^{1+\alpha_{0}}\left(\omega_{e}\right) \subset$ $W^{2, p}\left(\omega_{e}\right)$ with $p=2 /\left(2-\alpha_{0}\right)$; indeed, one calculates $\left\|S_{k}^{e}\right\|_{H^{1+\alpha_{0}}\left(\omega_{e}\right)} \lesssim\left\|S_{k}^{e}\right\|_{W^{2, p}\left(\omega_{e}\right)} \lesssim$ $k^{2\left(\alpha_{0}-\alpha\right) /\left(2-\alpha_{0}\right)}$.

Lemma 4.2 For any $w \in V_{1}^{1+\alpha_{0}}(\omega) \cap \stackrel{\circ}{V_{1}^{1}}(\omega)$, there holds:

$$
\left\|w-P_{h} w\right\|_{1,1} \lesssim h^{\alpha_{0}}\|w\|_{1+\alpha_{0}, 1}, \quad\left\|w-P_{h} w\right\|_{0,1} \lesssim h^{1+\alpha_{0}}\|w\|_{1+\alpha_{0}, 1} .
$$

Proof: Assume first that $w \in V_{1}^{2}(\omega) \cap \stackrel{\circ}{V_{1}^{1}}(\omega)$. From [8, Thm 4.2], we know

$$
h^{-1}\left\|w-P_{h} w\right\|_{0,1}+\left\|w-P_{h} w\right\|_{1,1} \lesssim h^{s-1}\|w\|_{s, 1} \quad \text { for } s=1,2 ;
$$

and, from [9, Prop. 1.e.1], that $V_{1}^{1+\alpha_{0}}(\omega)$ is the interpolate of order $\alpha_{0}$ between $V_{1}^{1}(\omega)$ and $V_{1}^{2}(\omega)$. Hence the two estimates in (50) by a standard interpolation argument. Then one generalises to $w \in V_{1}^{1+\alpha_{0}}(\omega) \cap \stackrel{\circ}{V_{1}^{1}}(\omega)$ by Lemma 4.1 .

Lemma 4.3 For $w \in H_{1}^{2}(\omega)$, there holds:

$$
\left\|w-\Pi_{h} w\right\|_{1-\sigma, 1} \approx\left|w-\Pi_{h} w\right|_{1-\sigma, 1} \lesssim h^{1+\sigma}|w|_{2,1} .
$$

for any $\sigma \in[0,1]$.

Proof: It stems from [18, Prop. 6.1] or [8, Prop. 4.1] that

$$
h^{-1}\left\|w-\Pi_{h} w\right\|_{0,1}+\left|w-\Pi_{h} w\right|_{1,1} \lesssim h|w|_{2,1} .
$$

One concludes by interpolating in the scale $H_{1}^{s}(\omega)$. 


\section{Discrete formulation, SCM}

In $\S \$ 5.1$ to 5.3 , the superscript 2 in $p_{s}^{2}, \varphi_{s}^{2}, \delta^{2}$, etc. will generally be omitted.

\subsection{Approximation of the dual singular function $p_{s}^{2}$.}

We start from the decomposition (29). $\tilde{p}$ is characterised by the three conditions

$$
\tilde{p} \in V_{1}^{1}(\omega), \quad \tilde{p}=-p_{P} \text { on } \gamma, \quad-\Delta_{2} \tilde{p}=\Delta_{2} p_{P} \text { in } \omega .
$$

A direct calculation shows that, denoting $\phi^{\prime}=\phi+\phi_{0}$ (see Figure 11):

$$
\Delta_{2} p_{P}=-\frac{5 r}{a^{2}} \alpha \rho^{-\alpha-1} \sin \left(\alpha \phi+\phi^{\prime}\right) .
$$

This function is of $H^{-1}$ regularity near the reentrant edge, and smooth elsewhere, so it belongs to the dual of $\stackrel{\circ}{V}_{1}^{1}(\omega)$. However, it should be noticed that $\Delta_{2} p_{P}$ never belongs locally to $L^{2}\left(\omega_{e}\right)$. This phenomenon causes the local regularity of $\tilde{p}$ to be weaker than in the prismatic case, and dramatically deteriorates the convergence rate of the SCM.

This inconvenience can be overcome by enriching the principal part with the next term in the expansion of $p_{s}$ near the reentrant corner. To do so, we look for a function in $V_{1}^{1}(\omega)$, with a $V_{1}^{2}$ regularity near the axis, vanishing on $\partial \omega_{e} \cap \gamma$, and whose Laplacian $\Delta_{2}$ is locally "almost equal" to $\Delta_{2} p_{P}$. First, we look for a local variational solution of

$$
-\Delta Q=\alpha \rho^{-\alpha-1} \sin \left(\alpha \phi+\phi^{\prime}\right) \text { in } \omega_{e}, \quad Q=0 \text { on } \partial \omega_{e} \cap \gamma .
$$

By separation of variables, we obtain: $Q(\rho, \phi)=\frac{1}{2} \rho^{1-\alpha} \cos \phi^{\prime} \sin (\alpha \phi)$. As the exponent $1-\alpha>0, Q$ does belong to $H^{1}\left(\omega_{e}\right)$. To obtain the $V_{1}^{2}$ regularity near the axis, we have to multiply it by $(r / a)^{2}$. A simple calculation shows that:

$$
\begin{aligned}
\Delta_{2}\left[\left(\frac{r}{a}\right)^{2} Q\right]= & -\left(\frac{r}{a}\right)^{2} \alpha \rho^{-\alpha-1} \sin \left(\alpha \phi+\phi^{\prime}\right) \\
& +\frac{5 r}{2 a^{2}} \rho^{-\alpha}\left[\sin (\alpha \phi)-\alpha \cos \phi^{\prime} \sin \left(\alpha \phi+\phi^{\prime}\right)\right] .
\end{aligned}
$$

Hence, the new decomposition:

$$
p_{s}=p_{p}+\widehat{p}, \text { where: } p_{p} \stackrel{\text { def }}{=} p_{P}-\frac{5}{a}\left(\frac{r}{a}\right)^{2} Q=\left(\frac{r}{a}\right)^{2}\left[1-\frac{5 \rho}{2 a} \cos \phi^{\prime}\right] \rho^{-\alpha} \sin (\alpha \phi),
$$

enjoys the following properties. First, $\widehat{p}=\tilde{p}+\left(5 r^{2} / a^{3}\right) Q \in V_{1}^{1}(\omega)$ and it vanishes on $\partial \omega_{e} \cap \gamma$. Then, using

$$
\left(\frac{r}{a}\right)^{2}-\frac{r}{a}=\frac{r(r-a)}{a^{2}}=\frac{r}{a^{2}} \rho \cos \phi^{\prime}
$$

we obtain

$$
\vartheta_{p} \stackrel{\text { def }}{=} \Delta_{2} p_{p}=\frac{r}{a^{3}} \rho^{-\alpha}\left[-\frac{25}{2} \sin (\alpha \phi)+\frac{35}{2} \alpha \cos \phi^{\prime} \sin \left(\alpha \phi+\phi^{\prime}\right)\right] \in L_{1}^{2}(\omega) .
$$

As $-\Delta_{2} \widehat{p}=\vartheta_{p}$, we infer by localisation that $\widehat{p} \in H^{1+\alpha_{0}}\left(\omega_{e}\right)$. Elsewhere, the smoothness of $\vartheta_{p}$ implies that of $\widehat{p}$, so $\widehat{p} \in V_{1}^{1+\alpha_{0}}(\omega)$, and is $V_{1}^{2}$ near the axis. 
Now, we are ready to derive the FE approximation of $p_{s}$. The variable $\widehat{p}$ solves the variational problem: Find $\widehat{p} \in V_{1}^{1}(\omega)$ such that

$$
\widehat{p}=s \text { on } \partial \omega, \quad \text { and } \quad a_{2}(\widehat{p}, v)=\left(\vartheta_{p} \mid v\right) \quad \forall v \in \stackrel{\circ}{V_{1}^{1}}(\omega)
$$

Similarly to the prismatic case [12, §5.1], we introduce

- the boundary function $s$ which is equal to the trace of $-p_{p}$, hence is zero on the two sides that meet at the reentrant corner, and smooth elsewhere;

- the smooth extension $\tilde{s} \in H_{1}^{2}(\omega)$ of $s$ into $\omega$;

- the variable $p^{\circ}=\widehat{p}-\tilde{s}$.

In the variable $p^{\circ}$, the problem (154) reads: Find $p^{\circ} \in \stackrel{\circ}{V}_{1}^{1}(\omega)$ such that

$$
a_{2}\left(p^{\circ}, v\right)=\left(\vartheta_{p} \mid v\right)-a_{2}(\tilde{s}, v) \quad \forall v \in \stackrel{\circ}{V_{1}^{1}}(\omega)
$$

and we have $p^{\circ} \in V_{1}^{1+\alpha_{0}}(\omega) \cap \stackrel{\circ}{V_{1}^{1}}(\omega)$. Here, too, we approximate $\widehat{p}$ by $\widehat{p}^{h}=\Pi_{h} \tilde{s}+p_{h}^{\circ}$, and $p_{s}$ by $p_{s}^{h}=p_{p}+\Pi_{h} \tilde{s}+p_{h}^{\circ}$, where $p_{h}^{\circ}$ solves the approximate FE problem

$$
a_{2}\left(p_{h}^{\circ}, v_{h}\right)=\left(v_{p}^{h} \mid v_{h}\right)-a_{2}\left(\Pi_{h} \tilde{s}, v_{h}\right) \quad \forall v_{h} \in V_{\circ}^{h} .
$$

The notation $\left(\vartheta_{p}^{h} \mid v_{h}\right)$ stands for an approximation by a quadrature formula of the integral $\iint_{\omega} \vartheta_{p}(r, z) \bar{v}_{h}(r, z) r \mathrm{~d} r \mathrm{~d} z$, with $\vartheta_{p}(r, z)$ given by (53). As $\vartheta_{p} \in L_{1}^{2}(\omega)$, we can suppose that the error caused by this quadrature is bounded as

$$
\left|\left(\vartheta_{p}^{h}-\vartheta_{p} \mid w_{h}\right)\right| \leq C_{\mathbf{Q}}^{1} h^{\mathbf{q}_{1}}\left\|w_{h}\right\|_{1,1}, \quad \forall w_{h} \in V^{h}, \quad \text { for some } C_{\mathbf{Q}}^{1}>0 \text { and } \mathbf{q}_{1} \geq 1 .
$$

This can be done e.g. by using a sixth-order Gauss-Hammer formula [21, p. 201], with seven points inside each triangle, which does not require the unbounded value of $\vartheta_{p}$. Of course, if $w_{h}$ vanishes on $\gamma_{a}$, one can replace $\left\|w_{h}\right\|_{1,1}$ with the stronger norm $\left\|w_{h}\right\|_{(2)}$ in (57).

Lemma 5.1 Assume $\mathrm{q}_{1} \geq 2$; then we have:

$$
\left\|p_{s}-p_{s}^{h}\right\|_{1,1} \lesssim h^{\alpha_{0}}, \quad\left\|p_{s}-p_{s}^{h}\right\|_{0,1} \lesssim h^{2 \alpha_{0}}
$$

Proof: Subtracting (56) from (55) yields:

$$
a_{2}\left(p^{\circ}-p_{h}^{\circ}, v_{h}\right)=\left(\vartheta_{p}-\vartheta_{p}^{h} \mid v_{h}\right)-a_{2}\left(\tilde{s}-\Pi_{h} \tilde{s}, v_{h}\right) \quad \forall v_{h} \in V_{\circ}^{h} .
$$

With $v_{h}=p_{h}^{\circ}-w_{h}$, this implies:

$$
\left\|p^{\circ}-w_{h}\right\|_{(2)}^{2} \geq\left\|p^{\circ}-p_{h}^{\circ}\right\|_{(2)}^{2}+2 a_{2}\left(\tilde{s}-\Pi_{h} \tilde{s}, p_{h}^{\circ}-w_{h}\right)+2\left(\vartheta_{p}-\vartheta_{p}^{h} \mid p_{h}^{\circ}-w_{h}\right) .
$$


Now, we set $w_{h}=P_{h} p^{\circ}$. Using (57), we obtain

$$
\begin{aligned}
\left\|p^{\circ}-p_{h}^{\circ}\right\|_{(2)}^{2} \leq & \left\|p^{\circ}-P_{h} p^{\circ}\right\|_{(2)}^{2}+2\left\|\tilde{s}-\Pi_{h} \tilde{s}\right\|_{(2)}\left(\left\|p_{h}^{\circ}-p^{\circ}\right\|_{(2)}+\left\|p^{\circ}-P_{h} p^{\circ}\right\|_{(2)}\right) \\
& +C_{Q}^{1} h^{\mathrm{q}_{1}}\left(\left\|p_{h}^{\circ}-p^{\circ}\right\|_{(2)}+\left\|p^{\circ}-P_{h} p^{\circ}\right\|_{(2)}\right) .
\end{aligned}
$$

With the Young inequality, the above estimate becomes:

$$
\begin{aligned}
\left\|p^{\circ}-p_{h}^{\circ}\right\|_{(2)}^{2} \leq & \left\|p^{\circ}-P_{h} p^{\circ}\right\|_{(2)}^{2}+5\left\|\tilde{s}-\Pi_{h} \tilde{s}\right\|_{(2)}^{2}+\frac{1}{4}\left\|p_{h}^{\circ}-p^{\circ}\right\|_{(2)}^{2}+\left\|p^{\circ}-P_{h} p^{\circ}\right\|_{(2)}^{2} \\
& +\frac{C_{\mathrm{Q}}^{1}}{2}\left[\left(2 C_{\mathrm{Q}}^{1}+1\right) h^{2 \mathbf{q}_{1}}+\frac{\left\|p_{h}^{\circ}-p^{\circ}\right\|_{(2)}^{2}}{2 C_{\mathrm{Q}}^{1}}+\left\|p^{\circ}-P_{h} p^{\circ}\right\|_{(2)}^{2}\right] .
\end{aligned}
$$

Thanks to the equivalence of norms $\|\cdot\|_{(2)} \approx\|\cdot\|_{1,1}$, we are left with the estimate:

$$
\left\|p^{\circ}-p_{h}^{\circ}\right\|_{1,1}^{2} \lesssim\left\|p^{\circ}-P_{h} p^{\circ}\right\|_{1,1}^{2}+\left\|\tilde{s}-\Pi_{h} \tilde{s}\right\|_{1,1}^{2}+h^{2 q_{1}} .
$$

By [18, Prop. 6.1], there holds: $\left\|\tilde{s}-\Pi_{h} \tilde{s}\right\|_{1,1} \lesssim h|s|_{2,1}$; by Lemma 4.2. we have $\left\|p^{\circ}-P_{h} p^{\circ}\right\|_{1,1} \lesssim h^{\alpha_{0}}\left\|p^{\circ}\right\|_{1+\alpha_{0}, 1} . \quad s$ and $p^{\circ}$ depend only on the geometry of $\omega$ so all their norms can be seen as constants. Hence, as soon as $\mathrm{q}_{1} \geq 1$, there holds: $\left\|p^{\circ}-p_{h}^{\circ}\right\|_{1,1}^{2} \lesssim h^{2 \alpha_{0}}$. Finally:

$$
\left\|p_{s}-p_{s}^{h}\right\|_{1,1}=\left\|p^{\circ}+\tilde{s}-p_{h}^{\circ}-\Pi_{h} \tilde{s}\right\|_{1,1} \leq\left\|p^{\circ}-p_{h}^{\circ}\right\|_{1,1}+\left\|\tilde{s}-\Pi_{h} \tilde{s}\right\|_{1,1} \lesssim h^{\alpha_{0}} .
$$

The obtention of the $L_{1}^{2}$ norm estimate also follows the prismatic case closely. Here, we define $w$ as the variational solution in $\stackrel{\circ}{V}_{1}^{1}(\omega)$ to

$$
a_{2}(w, v)=\left(p^{\circ}-p_{h}^{\circ} \mid v\right), \quad \forall v \in \stackrel{\circ}{V_{1}^{1}}(\omega) .
$$

By elliptic theory [10, Thm II.4.8] we know $w \in H_{(2)}^{1+\alpha_{0}}(\omega)=V_{1}^{1+\alpha_{0}}(\omega)$ and $\|w\|_{1+\alpha_{0}, 1} \lesssim$ $\left\|p^{\circ}-p_{h}^{\circ}\right\|_{0,1}$. Its FE approximation $w_{h}$ solves

$$
a_{2}\left(w_{h}, v_{h}\right)=\left(p^{\circ}-p_{h}^{\circ} \mid v_{h}\right), \quad \forall v \in V_{\circ}^{h},
$$

so $\left\|w_{h}\right\|_{(2)} \lesssim\left\|p^{\circ}-p_{h}^{\circ}\right\|_{0,1}$; by using Céa's lemma and Lemma 4.2 we infer:

$$
\left\|w-w_{h}\right\|_{(2)} \lesssim h^{\alpha_{0}}\|w\|_{1+\alpha_{0}} \lesssim h^{\alpha_{0}}\left\|p^{\circ}-p_{h}^{\circ}\right\|_{0,1} .
$$

Then, using successively (60) and (58), we obtain

$$
\left\|p^{\circ}-p_{h}^{\circ}\right\|_{0,1}^{2}=a_{2}\left(w-w_{h}, p^{\circ}-p_{h}^{\circ}\right)+\left(\vartheta_{p}-\vartheta_{p}^{h} \mid w_{h}\right)+a_{2}\left(\tilde{s}-\Pi_{h} \tilde{s}, w-w_{h}\right)-a_{2}\left(\tilde{s}-\Pi_{h} \tilde{s}, w\right) .
$$

This is bounded by the Cauchy inequality and (57), as well as the duality argument in the scale $V_{1}^{s}(\omega)$ :

$$
\begin{aligned}
\left\|p^{\circ}-p_{h}^{\circ}\right\|_{0,1}^{2} \lesssim & \left\|p^{\circ}-p_{h}^{\circ}\right\|_{(2)}\left\|w-w_{h}\right\|_{(2)}+h^{\mathrm{q}_{1}}\left\|w_{h}\right\|_{(2)} \\
& +\left\|\tilde{s}-\Pi_{h} \tilde{s}\right\|_{(2)}\left\|w-w_{h}\right\|_{(2)}+\left\|\tilde{s}-\Pi_{h} \tilde{s}\right\|_{1-\alpha_{0}, 1}\|w\|_{1+\alpha_{0}, 1} \\
\lesssim & \left\|p^{\circ}-p_{h}^{\circ}\right\|_{0,1}\left\{h^{\alpha_{0}} h^{\alpha_{0}}+h^{\mathrm{q}_{1}}+h|\tilde{s}|_{2,1} \times h^{\alpha_{0}}+h^{1+\alpha_{0}}|\tilde{s}|_{2,1} \times h^{0}\right\}
\end{aligned}
$$


where we have made use of [18, Prop. 6.1] and our Lemma 4.3. In order to get the $h^{2 \alpha_{0}}$ estimate, we have to suppose $q_{1} \geq 2$. Using once more (51), we obtain:

$$
\left\|p_{s}-p_{s}^{h}\right\|_{0,1} \leq\left\|p^{\circ}-p_{h}^{\circ}\right\|_{0,1}+\left\|\tilde{s}-\Pi_{h} \tilde{s}\right\|_{0,1} \lesssim h^{2 \alpha_{0}} .
$$

We are also confronted with the task of approximating $q_{s} \stackrel{\text { def }}{=} p_{s} / r^{2}$. The scalar product $\left(z^{k} \mid q_{s}\right)$ (see (74) below) is needed to compute the singularity coefficient. However, since $p_{s}^{h}$ is just element-wise linear, it is locally proportional to $r$ in the triangles which have one or two vertices on the axis; so $q_{s}^{h} \stackrel{\text { def }}{=} r^{-2} p_{s}^{h} \notin L_{1}^{2}(\omega)$. This is why we cannot hope to control any such thing as $\left\|q_{s}-q_{s}^{h}\right\|_{0,1}$.

Yet, thanks to the bounds (59) and (61) for $p_{s}^{h}-p_{s}$, we do have the weak estimates:

$$
\begin{aligned}
&\left|\left(q_{s}-q_{s}^{h} \mid v\right)\right| \lesssim h^{2 \alpha_{0}}\|v\|_{0,-3}, \quad \forall v \in L_{-3}^{2}(\omega), \\
& \text { resp. }\left|\left(q_{s}-q_{s}^{h} \mid v\right)\right| \lesssim h^{\alpha_{0}}\|v\|_{0,-1}, \quad \forall v \in L_{-1}^{2}(\omega) .
\end{aligned}
$$

\subsection{Approximation of the primal singular function $\varphi_{s}^{2}$.}

We start from (30), which is sufficient for obtaining error estimates similar to those of the prismatic case. Using (16), we see that $\tilde{\varphi}$, satisfying $\tilde{\varphi}=-\delta \varphi_{P}$ on $\partial \omega$, solves the variational problem:

$$
\begin{array}{ll} 
& a_{2}(\tilde{\varphi}, v)=\left(p_{s} \mid v\right)+\delta\left(\psi_{P} \mid v\right), \quad \forall v \in \stackrel{\circ}{V}_{1}^{1}(\omega), \\
\text { where: } \quad & \psi_{P} \stackrel{\text { def }}{=} \Delta_{2} \varphi_{P}=\frac{5 r}{a^{2}} \alpha \rho^{\alpha-1} \sin \left[(\alpha-1) \phi-\phi_{0}\right] .
\end{array}
$$

We propose the following finite element approximation of $\tilde{\varphi}$ in $V^{h}$ :

$$
\tilde{\varphi}_{h}=-\delta_{h} \pi_{h} \varphi_{P}+\varphi_{h}^{0},
$$

where: $\pi_{h} \varphi_{P}$ is a simple lifting of the boundary condition, cf. [12, Eq. (40)]; the singularity coefficient $\delta_{h}$ is computed using $\delta_{h}=\frac{1}{a \pi} \iint_{\omega}\left(p_{s}^{h}\right)^{2} r \mathrm{~d} \omega$; and $\varphi_{h}^{0} \in V_{\circ}^{h}$ is such that $\tilde{\varphi}_{h}$ is solution to the problem:

$$
a_{2}\left(\tilde{\varphi}_{h}, v_{h}\right)=\left(p_{s}^{h} \mid v_{h}\right)+\delta_{h}\left(\psi_{P}^{h} \mid v_{h}\right), \quad \forall v_{h} \in V_{\circ}^{h} .
$$

Like above, we assume that the quadrature formula denoted by $\left(\psi_{P}^{h} \mid v_{h}\right)$ satisfies:

$$
\left|\left(\psi_{P}^{h}-\psi_{P} \mid w_{h}\right)\right| \leq C_{\mathbf{Q}}^{2} h^{\mathbf{q}_{2}}\left\|w_{h}\right\|_{1,1}, \quad \forall w_{h} \in V^{h}, \quad \text { for some } C_{\mathbf{Q}}^{2}>0 \text { and } \mathbf{q}_{2} \geq 1,
$$

where one can replace $\left\|w_{h}\right\|_{1,1}$ with $\left\|w_{h}\right\|_{(2)}$ if $\left.w_{h}\right|_{\gamma_{a}}=0$. Then, we propose to compute the finite element approximation of $\varphi_{s}$ as:

$$
\varphi_{s}^{h}=\tilde{\varphi}_{h}+\delta_{h} \varphi_{P} .
$$


Lemma 5.2 The following error estimates hold:

$$
\left\|\varphi_{s}-\varphi_{s}^{h}\right\|_{1,1} \lesssim h, \quad\left\|\varphi_{s}-\varphi_{s}^{h}\right\|_{(k)} \lesssim k h .
$$

Proof: We follow the lines of the proof of Lemma 5.2 of the companion paper [12, taking care of the extra error generated by the quadrature. Subtracting (66) from (64), we obtain

$a_{2}\left(\tilde{\varphi}-\tilde{\varphi}_{h}, v_{h}\right)=\left(p_{s}-p_{s}^{h} \mid v_{h}\right)+\left(\delta-\delta_{h}\right)\left(\psi_{P} \mid v_{h}\right)+\delta_{h}\left(\psi_{P}-\psi_{P}^{h} \mid v_{h}\right), \quad \forall v_{h} \in V_{\circ}^{h}$.

So, for any $w_{h} \in V^{h}$ satisfying $w_{h}-\tilde{\varphi}_{h} \in V_{\circ}^{h}$ :

$$
\begin{aligned}
\left\|\tilde{\varphi}-\tilde{\varphi}_{h}\right\|_{(2)}^{2} \leq & \left\|\tilde{\varphi}-w_{h}\right\|_{(2)}^{2}+2\left\{\left\|p_{s}-p_{s}^{h}\right\|_{0,1}+\left|\delta-\delta_{h}\right|\left\|\psi_{P}\right\|_{0,1}\right. \\
& \left.+\left|\delta_{h}\right| C_{\mathrm{Q}}^{2} h^{\mathrm{q}_{2}}\left(\left\|\tilde{\varphi}-\tilde{\varphi}_{h}\right\|_{(2)}+\left\|\tilde{\varphi}-w_{h}\right\|_{(2)}\right)\right\} \\
\leq & 2\left\|\tilde{\varphi}-w_{h}\right\|_{(2)}^{2}+\frac{1}{2}\left\|\tilde{\varphi}-\tilde{\varphi}_{h}\right\|_{(2)}^{2} \\
& +C\left(\left\|p_{s}-p_{s}^{h}\right\|_{0,1}^{2}+\left|\delta-\delta_{h}\right|^{2}\left\|\psi_{P}\right\|_{0,1}^{2}+\left|\delta_{h}\right|^{2} h^{2 \mathrm{q}_{2}}\right) .
\end{aligned}
$$

But $\left\|\psi_{P}\right\|_{0,1}$ is a constant of the domain, and the error on the singularity coefficient is bounded as

$$
\left|\delta-\delta_{h}\right|=\frac{1}{a \pi}\left|\left\|p_{s}\right\|_{0,1}^{2}-\left\|p_{s}^{h}\right\|_{0,1}^{2}\right| \lesssim\left\|p_{s}-p_{s}^{h}\right\|_{0,1} \lesssim h^{2 \alpha_{0}}
$$

hence $\left|\delta_{h}\right| \approx 1$. With Lemma 5.1 (68) becomes

$$
\left\|\tilde{\varphi}-\tilde{\varphi}_{h}\right\|_{(2)}^{2} \lesssim\left\|\tilde{\varphi}-w_{h}\right\|_{(2)}^{2}+h^{4 \alpha_{0}}+h^{2 \mathrm{q}_{2}}
$$

To obtain an $h^{1}$ estimate, it is thus sufficient to assume $q_{2} \geq 1$. We then derive from (70) that, with $w_{h}=\delta_{h} \Pi_{h} \tilde{\varphi} / \delta$

$$
\left\|\tilde{\varphi}-\tilde{\varphi}_{h}\right\|_{(2)}^{2} \lesssim h^{4 \alpha_{0}}+|\delta|^{-2}\left\{\left|\delta-\delta_{h}\right|^{2}|\tilde{\varphi}|_{1}^{2}+\left|\delta_{h}\right|^{2}\left\|\tilde{\varphi}-\Pi_{h} \tilde{\varphi}\right\|_{(2)}^{2}\right\} .
$$

As $\tilde{\varphi} \in H_{1}^{2}(\omega)$, we have from [18, Prop. 6.1]: $\left\|\tilde{\varphi}-\Pi_{h} \tilde{\varphi}\right\|_{(2)} \approx\left\|\tilde{\varphi}-\Pi_{h} \tilde{\varphi}\right\|_{1,1} \lesssim h|\tilde{\varphi}|_{2,1}$, which with (69) gives: $\left\|\tilde{\varphi}-\tilde{\varphi}_{h}\right\|_{1,1} \approx\left\|\tilde{\varphi}-\tilde{\varphi}_{h}\right\|_{(2)} \lesssim h$, and finally:

$$
\left\|\varphi_{s}-\varphi_{s}^{h}\right\|_{1,1} \leq\left\|\tilde{\varphi}-\tilde{\varphi}_{h}\right\|_{1,1}+\left|\delta-\delta_{h}\right|\left\|\varphi_{P}\right\|_{1,1} \lesssim h .
$$

Finally, the estimate on $\left\|\varphi_{s}-\varphi_{s}^{h}\right\|_{(k)}$ follows from

$$
\left\|\varphi_{s}-\varphi_{s}^{h}\right\|_{(k)}^{2}=\left\|\varphi_{s}-\varphi_{s}^{h}\right\|_{(2)}^{2}+\mu\left\|\varphi_{s}-\varphi_{s}^{h}\right\|_{0,-1}^{2} \leq\left(1+\frac{\mu}{4}\right)\left\|\varphi_{s}-\varphi_{s}^{h}\right\|_{(2)}^{2} \lesssim k^{2} h^{2} .
$$




\subsection{Approximation of $\tilde{u}^{k}$ and $c_{k}$ in decomposition (17), for} $|k| \geq 2$.

Noting that $\tilde{u}^{k}$ and $c_{k}$ solve the coupled system (26] 27), it seems natural to formulate their finite element approximations as follows:

Find $\tilde{u}_{h}^{k} \in V_{\circ}^{h}$ and $c_{k}^{h} \in \mathbb{R}^{1}$ such that:

$$
\begin{aligned}
& a_{k}\left(\tilde{u}_{h}^{k}, v_{h}\right)+c_{k}^{h} a_{k}\left(\varphi_{s}^{h}, v_{h}\right)=\left(f^{k} \mid v_{h}\right) \quad \forall v_{h} \in V_{\circ}^{h}, \\
& \left(\left\|p_{s}^{h}\right\|_{0,1}^{2}+\mu\left[\left|\varphi_{s}^{h}\right|_{1,-1}^{2}+2\left\|\varphi_{s}^{h}\right\|_{0,-3}^{2}\right]\right) c_{k}^{h}+\mu\left(\tilde{u}_{h}^{k} \mid p_{s}^{h}\right)=\left(f^{k} \mid p_{s}^{h}\right) .
\end{aligned}
$$

However, like any function in $V_{\circ}^{h}, \varphi_{s}^{h}$ does not necessarily belong to $H_{-1}^{1}(\omega)$ or $L_{-3}^{2}(\omega)$. This is no serious problem: like in the prismatic case [12, §5.3], we shall rather discretise the representation formula (28), which we rewrite as follows:

$$
c_{k}=\frac{1}{\left\|p_{s}\right\|_{0,1}^{2}}\left[\left(f^{k} \mid p_{s}\right)-\mu\left(z^{k} \mid q_{s}\right)\right]
$$

where $q_{s}=p_{s} / r^{2}$ and $z_{k}=A_{k}^{-1} f^{k} \in \stackrel{\circ}{V_{1}^{1}}(\omega)$ solves

$$
a_{k}\left(z^{k}, v\right)=\left(f^{k} \mid v\right), \quad \forall v \in \stackrel{\circ}{V_{1}^{1}}(\omega) .
$$

So, we state the

SCM Algorithm for finding $\tilde{u}_{h}^{k} \in V_{\circ}^{h}$ and $c_{k}^{h} \in \mathbb{R}^{1}$.

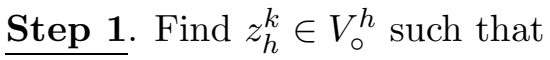

$$
a_{k}\left(z_{h}^{k}, v\right)=\left(f^{k} \mid v\right) \quad \forall v \in V_{\circ}^{h} .
$$

Compute $c_{k}^{h}$ as follows:

$$
c_{k}^{h}=\frac{1}{\left\|p_{s}^{h}\right\|_{0,1}^{2}}\left[\left(f^{k} \mid p_{s}^{h}\right)-\mu\left(z_{h}^{k} \mid q_{s}^{h}\right)\right], \quad \text { if } \quad k<C^{\star} h^{-\frac{1}{2-\alpha_{0}}}
$$

for some fixed constant $C^{\star}$, and

$$
c_{k}^{h}=0 \quad \text { if } \quad k \geq C^{\star} h^{-\frac{1}{2-\alpha_{0}}} .
$$

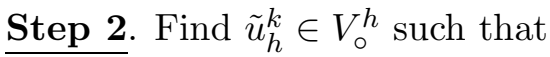

$$
a_{k}\left(\tilde{u}_{h}^{k}, v\right)+c_{k}^{h} a_{k}\left(\varphi_{s}^{h}, v\right)=\left(f^{k} \mid v\right) \quad \forall v \in V_{\circ}^{h} .
$$

Lemma 5.3 For the solution $z^{k}$ to the problem (75) and its piecewise linear finite element approximation $z_{h}^{k}$ in (76), we have the following error estimates

$$
\begin{aligned}
\left\|z^{k}-z_{h}^{k}\right\|_{0,-1} & \lesssim k^{-2}\left\|f^{k}\right\|_{0,1}, \\
\left\|z^{k}-z_{h}^{k}\right\|_{0,-1} & \lesssim k^{-1}\left[h^{\alpha_{0}} k^{\alpha_{0}-1}+h\right]\left\|f^{k}\right\|_{0,1}, \\
\left\|z^{k}-z_{h}^{k}\right\|_{0,1} & \lesssim\left[h^{2 \alpha_{0}} k^{2\left(\alpha_{0}-1\right)}+h^{2}\right]\left\|f^{k}\right\|_{0,1},
\end{aligned}
$$


while for the coefficient $c_{k}$ in (74) and its approximation $c_{k}^{h}$ in (77), we have

$$
\left|c_{k}-c_{k}^{h}\right| \lesssim\left(h^{2 \alpha_{0}} k^{2 \alpha_{0}}+h^{2} k^{2}\right)\left\|f^{k}\right\|_{0,1} .
$$

Proof: It follows from (75) and (76) that

$$
a_{k}\left(z^{k}-z_{h}^{k}, z^{k}-z_{h}^{k}\right)=a_{k}\left(z^{k}, z^{k}-z_{h}^{k}\right)=\left(f^{k} \mid z^{k}-z_{h}^{k}\right) .
$$

This implies

$$
\left|z^{k}-z_{h}^{k}\right|_{1,1}^{2}+k^{2}\left\|z^{k}-z_{h}^{k}\right\|_{0,-1}^{2} \leq\left\|f^{k}\right\|_{0,1}\left\|z^{k}-z_{h}^{k}\right\|_{0,1} \leq r_{\max }\left\|f^{k}\right\|_{0,1}\left\|z^{k}-z_{h}^{k}\right\|_{0,-1},
$$

hence (80). Then, using Céa's lemma, Lemma 4.2. Thm 7.1 of [18, and the bounds (20) and (23), we obtain another estimate:

$$
\begin{aligned}
\left\|z^{k}-z_{h}^{k}\right\|_{(k)}^{2} & \leq\left\|z^{k}-P_{h} z^{k}\right\|_{(k)}^{2} \\
& =\left\|z^{k}-P_{h} z^{k}\right\|_{1,1}^{2}+\left(k^{2}-1\right)\left\|z^{k}-P_{h} z^{k}\right\|_{0,-1}^{2} \\
& \lesssim h^{2 \alpha_{0}}\left|z^{k}\right|_{1+\alpha_{0}, 1}^{2}+\left(k^{2}-1\right) h^{2}\left|z^{k}\right|_{1,-1}^{2} \\
& \lesssim\left[h^{2 \alpha_{0}} k^{2\left(\alpha_{0}-1\right)}+h^{2}\right]\left\|f^{k}\right\|_{0,1}^{2}
\end{aligned}
$$

Of course, a similar bound holds for any $g \in L_{1}^{2}(\omega), w=A_{k}^{-1} g$ and $P_{h} w$. Thus, the estimate (82) follows from a duality argument like in [12. Lemma 5.3]. Moreover, we obtain (81) thanks to the bound: $\|\cdot\|_{0,-1}^{2} \leq k^{-2}\|\cdot\|_{(k)}^{2}$.

To obtain the estimate (83), we subtract (74) from (77) to obtain

$$
c_{k}-c_{k}^{h}=\left\{\frac{\left(f^{k} \mid p_{s}\right)}{\left\|p_{s}\right\|_{0,1}^{2}}-\frac{\left(f^{k} \mid p_{s}^{h}\right)}{\left\|p_{s}^{h}\right\|_{0,1}^{2}}\right\}+\mu\left\{\frac{\left(z_{h}^{k} \mid q_{s}^{h}\right)}{\left\|p_{s}^{h}\right\|_{0,1}^{2}}-\frac{\left(z^{k} \mid q_{s}\right)}{\left\|p_{s}\right\|_{0,1}^{2}}\right\} \stackrel{\text { def }}{=} I_{1}+I_{2} .
$$

We bound $I_{1}$ by Lemma 5.1 $\left|I_{1}\right| \lesssim h^{2 \alpha_{0}}\left\|f^{k}\right\|_{0,1}$. As for $I_{2}$, it is zero when $\mu=0$; otherwise we rewrite it as follows:

$$
\begin{aligned}
\frac{I_{2}}{\mu}= & \frac{1}{\left\|p_{s}^{h}\right\|_{0,1}^{2}}\left\{\left(z_{h}^{k}-z^{k} \mid q_{s}\right)+\left(z_{h}^{k}-z^{k} \mid q_{s}^{h}-q_{s}\right)+\left(z^{k} \mid q_{s}^{h}-q_{s}\right)\right\} \\
& +\left(z^{k} \mid q_{s}\right)\left\{\frac{1}{\left\|p_{s}^{h}\right\|_{0,1}^{2}}-\frac{1}{\left\|p_{s}\right\|_{0,1}^{2}}\right\} \\
\stackrel{\text { def }}{=} & J_{2}^{1}+J_{2}^{2}+J_{2}^{3}+J_{2}^{4} .
\end{aligned}
$$

Then, recalling that $\left\|q_{s}\right\|$ is constant, we estimate:

- $\left|J_{2}^{1}\right| \lesssim\left\|z_{h}^{k}-z^{k}\right\|_{0,1} \lesssim\left[h^{2 \alpha_{0}} k^{2\left(\alpha_{0}-1\right)}+h^{2}\right]\left\|f^{k}\right\|_{0,1}$ by (82).

- $\left|J_{2}^{2}\right| \lesssim h^{\alpha_{0}}\left\|z_{h}^{k}-z^{k}\right\|_{0,-1} \lesssim\left[h^{2 \alpha_{0}} k^{\alpha_{0}-2}+h^{1+\alpha_{0}} k^{-1}\right]\left\|f^{k}\right\|_{0,1}$ by (63) and (81). 
- $\left|J_{2}^{3}\right| \lesssim h^{2 \alpha_{0}}\left\|z^{k}\right\|_{0,-3} \lesssim h^{2 \alpha_{0}} k^{-2}\left\|f^{k}\right\|_{0,1}$ by (62) and (20).

- $\left|J_{2}^{4}\right| \lesssim h^{2 \alpha_{0}}\left\|z^{k}\right\|_{0,1} \leq h^{2 \alpha_{0}} r_{\max }\left\|z^{k}\right\|_{0,-1} \lesssim h^{2 \alpha_{0}} k^{-2}\left\|f^{k}\right\|_{0,1}$ by (61) and (19)).

Summarising, we obtain

$$
\begin{aligned}
\left|c_{k}-c_{k}^{h}\right| & \leq\left|I_{1}\right|+\mu\left\{\left|J_{2}^{1}\right|+\left|J_{2}^{2}\right|+\left|J_{2}^{3}\right|+\left|J_{2}^{4}\right|\right\} \\
& \lesssim\left(h^{2 \alpha_{0}}+h^{2 \alpha_{0}} k^{\alpha_{0}}+h^{2 \alpha_{0}} k^{2 \alpha_{0}}+h^{1+\alpha_{0}} k+h^{2} k^{2}\right)\left\|f^{k}\right\|_{0,1} .
\end{aligned}
$$

The estimate (83) then follows by remarking that the first, second and fourth terms in the bracket are negligible with respect to the third.

Now, we observe that the formula (79) for computing $\tilde{u}_{h}^{k}$, as well as the SCM reconstruction formula for $u_{h}^{k}$

$$
u_{h}^{k}=\tilde{u}_{h}^{k}+c_{k}^{h} \varphi_{s}^{h}=\tilde{u}_{h}^{k}+c_{k}^{h}\left(\tilde{\varphi}_{h}+\delta_{h} \varphi_{P}\right) .
$$

are formally identical to their prismatic counterparts (cf. the SCM algorithm of [12, $\S 5.3]$ ); and the "building blocks" $c_{k}^{h}$ and $\varphi_{s}^{h}$ also satisfy estimates similar to those of the prismatic case. Indeed, under the assumption $k<C^{\star} h^{-\frac{1}{2-\alpha_{0}}}$, both terms within the bracket in (83) are negligible with respect to $h k$. Hence the following two results, whose proofs closely parallel that of Lemma 5.4 and Theorem 5.1 in [12, with the same kind of adaptations (use of weighted norms, $P_{h}$ and Lemma 4.2) as usual.

Lemma 5.4 The following error estimate holds:

$$
\left\|\tilde{u}^{k}-\tilde{u}_{h}^{k}\right\|_{(k)}^{2} \lesssim k\left(h^{2}\left(1+k^{2} h^{2}\right)\left\|f^{k}\right\|_{0,1}^{2}+\left|c_{k}-c_{k}^{h}\right|^{2}\right) .
$$

Theorem 5.1 Let $u^{k}$ be the solution to the equation (20) and $u_{h}^{k}$ be its finite element approximation given in (84). Then the following error estimate holds:

$$
\left\|u^{k}-u_{h}^{k}\right\|_{(k)} \lesssim k^{2} h\left\|f^{k}\right\|_{0,1}
$$

\subsection{Approximation of the singular functions for the modes $|k|=0,1$.}

The FE approximation of these functions has been exposed in [5, $\S \S 4.1$ and 4.2]. (In that work, the Laplacians $\Delta_{0}$ and $\Delta_{1}$ are respectively called $\Delta$ and $\Delta^{\prime}$ ). We keep this method, with the following modification. The dual singular functions associated to the reentrant edge undergo the same inconvenience as $p_{s}^{2}$, namely, the Laplacian of the principal parts as defined in [5] do not belong to $L_{1}^{2}(\omega)$. Hence we must enrich them, just as we did for $p_{P}^{2}$, in order to preserve the convergence rate. Calculating like in 5.1, we obtain the following decompositions:

$$
\begin{gathered}
p_{s}^{1}=p_{p}^{1}+\widehat{p}^{1}, \quad p_{p}^{1} \stackrel{\text { def }}{=} \rho^{-\alpha} \sin (\alpha \phi) \frac{r}{a}\left[1-\frac{3 \rho}{2 a} \cos \phi^{\prime}\right], \quad \widehat{p}^{1} \in V_{1}^{1}(\omega) ; \\
p_{s}^{0, e}=p_{p}^{0, e}+\widehat{p}^{0, e}, \quad p_{p}^{0, e} \stackrel{\text { def }}{=} \rho^{-\alpha} \sin (\alpha \phi)\left[1-\frac{\rho}{2 a} \cos \phi^{\prime}\right], \quad \widehat{p}^{0, e} \in H_{1}^{1}(\omega) .
\end{gathered}
$$


The Laplacians of the principal parts are:

$$
\begin{aligned}
\vartheta_{p}^{1} \stackrel{\text { def }}{=} \Delta_{1} p_{p}^{1} & =\frac{1}{a^{2}} \rho^{-\alpha}\left[-\frac{9}{2} \sin (\alpha \phi)+\frac{15}{2} \alpha \cos \phi^{\prime} \sin \left(\alpha \phi+\phi^{\prime}\right)\right] \in L_{1}^{2}(\omega) ; \\
\vartheta_{p}^{0, e} \stackrel{\text { def }}{=} \Delta_{0} p_{p}^{0, e} & =\frac{1}{a r} \rho^{-\alpha}\left[-\frac{1}{2} \sin (\alpha \phi)+\frac{3}{2} \alpha \cos \phi^{\prime} \sin \left(\alpha \phi+\phi^{\prime}\right)\right] \in L_{1}^{2}(\omega) .
\end{aligned}
$$

Then we proceed like in $\$ 5.1$ to obtain:

$$
\begin{gathered}
\left\||| p_{s}^{1}-p_{s}^{1 ; h}||_{1,1} \lesssim h^{\alpha_{0}}, \quad\right\| p_{s}^{1}-p_{s}^{1 ; h} \|_{0,1} \lesssim h^{2 \alpha_{0}} \\
\left|p_{s}^{0, e}-p_{s}^{0, e ; h}\right|_{1,1} \lesssim h^{\alpha_{1}}, \quad\left\|p_{s}^{0, e}-p_{s}^{0, e ; h}\right\|_{0,1} \lesssim h^{2 \alpha_{1}}
\end{gathered}
$$

However, for the primal edge singular functions, the method of [5] yields the desired convergence rate. We just recall the decompositions:

$$
\varphi_{s}^{k}=\tilde{\varphi}^{k}+\delta^{k} \varphi_{P}^{k}, \quad \tilde{\varphi}^{k} \in H_{1}^{2}(\omega) \cap H_{(k)}^{1}(\omega), \quad \varphi_{P}^{k}=\left(\frac{r}{a}\right)^{k} \rho^{\alpha} \sin (\alpha \phi),
$$

as well as the Laplacians of the principal parts:

$$
\psi_{P}^{k} \stackrel{\text { def }}{=} \Delta_{k} \varphi_{P}^{k}=\frac{(k+1)(k+2)}{2 a^{k} r^{1-k}} \alpha \rho^{\alpha-1} \sin \left[(\alpha-1) \phi-\phi_{0}\right] .
$$

The line of proof already exposed in $\$ 5.2$ then easily leads to the error estimates:

$$
\left\|\varphi_{s}^{1}-\varphi_{s}^{1 ; h}\right\|_{1,1} \lesssim h \quad \text { and } \quad\left|\varphi_{s}^{0, e}-\varphi_{s}^{0, e ; h}\right|_{1,1} \lesssim h .
$$

Now, as far as the conical point singularities are concerned, the method appears very similar to that of [12, $\S \S 5.1$ and 5.2] since the principal parts $p_{P}^{0, c}$ and $\varphi_{P}^{0, c}$ have a vanishing Laplacian $\Delta_{0}$. So, mutatis mutandis, we get the error estimates:

$$
\left|p_{s}^{0, c}-p_{s}^{0, c ; h}\right|_{1,1} \lesssim h^{\alpha_{1}}, \quad\left\|p_{s}^{0, c}-p_{s}^{0, c ; h}\right\|_{0,1} \lesssim h^{2 \alpha_{1}}, \quad\left|\varphi_{s}^{0, c}-\varphi_{s}^{0, c ; h}\right|_{1,1} \lesssim h .
$$

Remark 5.1 Thanks to the asympotic expansions [1, Eq. 8.7.1] of the Legendre function, it is possible to compute the function $P_{\nu}(\cos \phi)$ with an arbitrary precision. Thus, one can compute once and for all the singularity exponent $\nu$ and the integral in 40 ) with an accuracy equal to the machine precision. All this guarantees that the errors due to the approximation of the conical singular functions will be negligible before the FE discretisation error.

\subsection{Approximation of $\tilde{u}^{k}$ and $c_{k}$, for $|k| \leq 1$.}

As the representation formulae (33) and (46) for the singularity coefficients of these modes are rather standard, one can use the simple discrete versions:

$$
c_{ \pm 1}^{h}=\frac{\left(f^{ \pm 1} \mid p_{s}^{1 ; h}\right)}{\left\|p_{s}^{1 ; h}\right\|_{0,1}^{2}}, \quad c_{0, j}^{h}=\frac{\left(f^{0} \mid p_{s}^{0, j ; h}\right)}{\left\|p_{s}^{0, j ; h}\right\|_{0,1}^{2}} .
$$


Similarly, we will approximate the regular parts $\tilde{u}^{k},|k| \leq 1$, by $\tilde{u}_{h}^{1}, \tilde{u}_{h}^{-1} \in V_{\circ}^{h}$, and $\tilde{u}_{h}^{0} \in V_{\diamond}^{h}$ such that

$$
\begin{array}{ll}
k= \pm 1: & a_{1}\left(\tilde{u}_{h}^{k}, v_{h}\right)+c_{k}^{h} a_{1}\left(\varphi_{s}^{1, h}, v_{h}\right)=\left(f^{k} \mid v_{h}\right), \quad \forall v_{h} \in V_{\circ}^{h}, \\
k=0: & a_{0}\left(\tilde{u}_{h}^{0}, v_{h}\right)+c_{0, e}^{h} a_{0}\left(\varphi_{s}^{0, e ; h}, v_{h}\right)+c_{0, c}^{h} a_{0}\left(\varphi_{s}^{0, c ; h}, v_{h}\right)=\left(f^{0} \mid v_{h}\right), \quad \forall v_{h} \in V_{\diamond}^{h} .
\end{array}
$$

Of course, we have the SCM reconstruction formulae:

$$
\begin{array}{rlrl}
k= \pm 1: & u_{h}^{k} & =\tilde{u}_{h}^{k}+c_{k}^{h} \varphi_{s}^{1 ; h}=\tilde{u}_{h}^{k}+c_{k}^{h}\left(\tilde{\varphi}_{h}^{1}+\delta_{h}^{1} \varphi_{P}^{1}\right) \\
k=0: \quad u_{h}^{0} & =\tilde{u}_{h}^{0}+c_{0, e}^{h} \varphi_{s}^{0, e ; h}+c_{0, c}^{h} \varphi_{s}^{0, c ; h} \\
& =\tilde{u}_{h}^{0}+c_{0, e}^{h}\left(\tilde{\varphi}_{h}^{0, e}+\delta_{h}^{0, e} \varphi_{P}^{0, e}\right)+c_{0, c}^{h}\left(\tilde{\varphi}_{h}^{0, c}+\delta_{h}^{0, c} \varphi_{P}^{0, c}\right) .
\end{array}
$$

The results of 5.4 then allow to conclude that:

$$
\begin{array}{ll}
k= \pm 1: \quad & \left|c_{k}-c_{k}^{h}\right| \lesssim h\left\|f^{k}\right\|_{0,1}, \quad\left\|\tilde{u}^{k}-\tilde{u}_{h}^{k}\right\|_{1,1} \lesssim h\left\|f^{k}\right\|_{0,1}, \\
& || \mid u^{k}-u_{h}^{k}\left\|_{1,1} \lesssim h\right\| f^{k} \|_{0,1} ; \\
k=0: \quad & \left|c_{0, j}^{h}-c_{0, j}\right| \lesssim h\left\|f^{0}\right\|_{0,1}, \quad\left|\tilde{u}^{0}-\tilde{u}_{h}^{0}\right|_{1,1} \lesssim h\left\|f^{0}\right\|_{0,1}, \\
& \left|u^{0}-u_{h}^{0}\right|_{1,1} \lesssim h\left\|f^{0}\right\|_{0,1} .
\end{array}
$$

\section{Fourier Singular Complement Method}

Let $u$ be the solution to the 3D problem (2), and $u^{k}$ its Fourier coefficients. From the previous Sections, we know that $u^{k}(r, z)$ solves the 2D problem (9), the weak formulation of the elliptic problem (7 7 , And, according to the mode $k$, one can decompose $u^{k}$ as (17), (32) or (45).

The result of Heinrich [15, Thm 5.2] can be straightforwardly extended to our domain with a sharp vertex.

Theorem 6.1 Let $f \in h^{2}(\Omega)$, and $u \in \stackrel{\circ}{H}^{1}(\Omega)$ be the solution to (2). Then:

$$
u(r, \theta, z)=\tilde{u}(r, \theta, z)+\gamma(\theta) \varphi_{s}^{2}(r, z)+c_{0}^{c} \varphi_{s}^{0, c}(r, z),
$$

with: $\tilde{u} \in H^{2}(\Omega) \cap \stackrel{\circ}{H^{1}}(\Omega)$, and $\gamma \in H^{2}\left(\mathbf{S}^{1}\right)$ is given by the formula:

$$
\gamma(\theta)=\frac{\delta^{0, e}}{\delta^{2}} c_{0}^{e}+\frac{\delta^{1}}{\delta^{2}} \sum_{k= \pm 1} c_{k} \mathrm{e}^{\mathrm{i} k \theta}+\sum_{|k| \geq 2} c_{k} \mathrm{e}^{\mathrm{i} k \theta}
$$

Like in the prismatic case (cf. [12], Remark 6.1), the hypothesis $f \in h^{2}(\Omega)$ is crucial: the lack of its satisfaction would prevent the convergence of $\gamma$ in a regular enough space, and hence that of the singular part of the solution in the natural space. 
We define the Fourier-SCM (FSCM) solution to (2) as follows:

$$
u_{h}^{[N]}=\sum_{k=-N}^{N} u_{h}^{k}(r, z) \mathrm{e}^{\mathrm{i} k \theta},
$$

where $u_{h}^{k}$ is the SCM solution to (9) algorithmically defined in \$5] The main result on this method is the following

Theorem 6.2 Assume $f \in h^{2}(\Omega)$. Then the following error estimate holds:

$$
\left|u-u_{h}^{[N]}\right|_{H^{1}(\Omega)} \lesssim\left(h+N^{-1}\right)\left\{\|f\|_{L^{2}(\Omega)}+\left\|\frac{\partial^{2} f}{\partial \theta^{2}}\right\|_{L^{2}(\Omega)}\right\} .
$$

Proof: Using the definition of $u_{h}^{[N]}$ and (6) we have

$$
\left|u-u_{h}^{[N]}\right|_{H^{1}(\Omega)}^{2}=\sum_{|k| \leq N}\left\|u^{k}-u_{h}^{k}\right\|_{(k)}^{2}+\sum_{|k|>N}\left\|u^{k}\right\|_{(k)}^{2} \stackrel{\text { def }}{=} E_{1}+E_{2} .
$$

Using (19), we estimate $E_{2}$ as:

$$
E_{2} \leq N^{-2} \sum_{|k|>N} k^{2}\left(\left|u^{k}\right|_{1,1}^{2}+k^{2}\left\|u^{k}\right\|_{0,-1}^{2}\right) \lesssim N^{-2} \sum_{|k|>N}\left\|f^{k}\right\|_{0,1}^{2} \leq N^{-2}\|f\|_{L^{2}(\Omega)}^{2} .
$$

As for $E_{1}$, we cut it into three parts, corresponding to $k=0,|k|=1$, and $2 \leq|k| \leq N$, which we bound respectively by (102) and (101) and (85):

$$
\begin{aligned}
E_{1} & \lesssim h^{2}\left\|f^{0}\right\|_{0,1}^{2}+h^{2}\left(\left\|f^{1}\right\|_{0,1}^{2}+\left\|f^{-1}\right\|_{0,1}^{2}\right)+h^{2} \sum_{2 \leq|k| \leq N} k^{4}\left\|f^{k}\right\|_{0,1}^{2} \\
& \lesssim h^{2}\left\{\|f\|_{L^{2}(\Omega)}+\left\|\frac{\partial^{2} f}{\partial \theta^{2}}\right\|_{L^{2}(\Omega)}\right\},
\end{aligned}
$$

where we have used Lemma 2.2 to bound the sum. Hence the result.

\section{Conclusion}

In this paper, we have proven that the FSCM for the Poisson equation achieves the optimal convergence rate for $\mathbb{P}_{1}$ finite elements and a datum of $L^{2}$-style regularity in the meridian directions. The same result also holds for the discretization of the Poisson problem with a homogeneous Neumann boundary condition, or with non-homogeneous boundary conditions, provided there exist sufficiently smooth liftings.

This result closely parallels that of the companion paper 12. The specificities of the axisymmetric geometry (namely, that the 2D problems are set in weighted Sobolev spaces, which moreover vary for the low-order Fourier modes before stabilising, and involve differential operators with non-constant coefficients) only cause technical difficulties. As far as the presence of conical vertices is concerned, its effect is no more than a finite-dimensional perturbation. Furthermore, it is no difficulty to consider the case of an axisymmetric domain $\Omega$ with several reentrant edges (i.e. $\omega$ with several off-axis reentrant corners) and/or several sharp vertices. 
As already mentioned, this paper is the second part of a three-part article [12, 13]. In [13, the FSCM is analysed from a numerical point of view (complexity, implementation issues, numerical experiments, etc.), and it is compared to other methods - in the axisymmetric case, to anisotropic mesh refinement techniques.

One can apply the same theoretical and numerical techniques to the fully axisymmetric heat or wave equations, with any $L^{2}$-smooth (in space) right-hand side. For these PDEs, the singular functions $p_{s}$ and $\varphi_{s}$ do not depend on the time-step. Finally, the results, can also be viewed as the first effort towards the discretization of electromagnetic fields in axisymmetric domains, with continuous numerical approximations, the importance of which is well-known, cf. 22. As a matter of fact, the SCM developed in 3, 4, 5] for fully axisymmetric electromagnetic computations can be generalized to arbitrary data, with the help of the results obtained here.

\section{References}

[1] Abramowitz, M., Stegun, I.A. (1965): Handbook of mathematical functions. Dover, New York.

[2] Assous, F., Degond, P., Heintzé, E., Raviart, P.-A., Segré, J. (1993): On a finite-element method for solving the three-dimensional Maxwell equations. J. Comput. Phys. 109, $222-237$.

[3] Assous, F., Ciarlet, Jr, P., Labrunie, S. (2002): Theoretical tools to solve the axisymmetric Maxwell equations. Math. Meth. Appl. Sci. 25, 49-78.

[4] Assous, F., Ciarlet, Jr, P., Labrunie, S. (2003): Solution of axisymmetric Maxwell equations. Math. Meth. Appl. Sci. 26, 861-896.

[5] Assous, F., Ciarlet, Jr, P., Labrunie, S., Segré, J. (2003): Numerical solution to the time-dependent Maxwell equations in axisymmetric singular domains: the Singular Complement Method. J. Comput. Phys. 191, 147-176.

[6] Assous, F., Ciarlet, Jr, P., Sonnendrücker, E. (1998): Resolution of the Maxwell equations in a domain with reentrant corners. Math. Model. Numer. Anal. 32, 359-389.

[7] Assous, F., Ciarlet, Jr., P., Segré, J. (2000): Numerical solution to the time-dependent Maxwell equations in two-dimensional singular domains: the Singular Complement Method. J. Comput. Phys. 161, 218-249.

[8] Belhachmi, Z., Bernardi, C., Deparis, S. (2003): Weighted Clément operator and application to the finite element discretization of the axisymmetric Stokes problem. Internal Report 03029, Laboratoire Jacques-Louis Lions, Université Pierre et Marie Curie.

[9] Bernardi, C., Dauge, M., Maday, Y. (1992): Polynomials in weighted Sobolev spaces: basics and trace liftings. Internal Report 92039, Laboratoire d'analyse numérique, Université Pierre et Marie Curie.

[10] Bernardi, C., Dauge, M., Maday, Y. (1999): Spectral methods for axisymmetric domains. Series in Applied Mathematics, Gauthier-Villars, Paris and North Holland, Amsterdam.

[11] Ciarlet, Jr, P., He, J. (2003): The singular complement method for 2D scalar problems. C. R. Acad. Sci. Paris, Ser. I 336 353-358.

[12] Ciarlet, Jr, P., Jung, B., Kaddouri, S., Labrunie, S., Zou, J. (2004): The Fourier Singular Complement Method for the Poisson problem. Part I: prismatic domains. To appear in Numer. Math. 
[13] Ciarlet, Jr, P., Jung, B., Kaddouri, S., Labrunie, S., Zou, J. (2004): The Fourier Singular Complement Method for the Poisson problem. Part III: implementation issues. In preparation.

[14] Grisvard, P. (1992): Singularities in boundary value problems. RMA 22, Masson, Paris.

[15] Heinrich, B. (1993): Singularity functions at axisymmetric edges and their representation by Fourier series. Math. Meth. Appl. Sci. 16, 837-854.

[16] Heinrich, B. (1996): The Fourier-finite element method for Poisson's equation in axisymmetric domains with edges. SIAM J. Numer. Anal. 33, 1885-1911.

[17] Heinrich, B., Nicaise S., Weber, B. (2000): Elliptic interface problems in axisymmetric domains II: Convergence analysis of the Fourier-finite element method. Adv. Math. Sci. Appl. 10 571-600.

[18] Mercier, B., Raugel, G. (1982): Résolution d'un problème aux limites dans un ouvert axisymétrique par éléments finis en $r, z$ et séries de Fourier en $\theta$. RAIRO Analyse numérique 4, 405-461.

[19] Nazarov, S.A., Plamenevsky, B.A. (1994): Elliptic problems in domains with piecewise smooth boundaries. De Gruyter Exp. Math. 13.

[20] Raugel, G. (1978): Résolution numérique de problèmes elliptiques dans des domaines avec coins. PhD Thesis, Rennes University, France.

[21] Zienkiewicz, O.C. (1977): The Finite Element Method. McGraw-Hill, London. 